\title{
DISTRIBUTED DETERMINISTIC ASYNCHRONOUS ALGORITHMS IN TIME-VARYING GRAPHS THROUGH DYKSTRA SPLITTING*
}

\author{
C.H. JEFFREY PANG ${ }^{\dagger}$
}

\begin{abstract}
Consider the setting where each vertex of a graph has a function, and communications can only occur between vertices connected by an edge. We wish to minimize the sum of these functions. For the case when each function is the sum of a strongly convex quadratic and a convex function, we propose a distributed version of Dykstra's algorithm. The computations to optimize the dual objective function can run asynchronously without a global clock, and in a distributed manner without a central controller. Convergence to the primal minimizer is deterministic instead of being probabilistic, and is guaranteed as long as in each cycle, the edges where two-way communications occur connects all vertices. We also look at an accelerated algorithm, and an algorithm for the case when the functions on the nodes are not strongly convex.
\end{abstract}

Key words. Distributed optimization, Averaged consensus, Dykstra's algorithm, time-varying graphs

AMS subject classifications. 68W15, 90C25, 90C30, 65K05

1. Introduction. Let $\mathcal{G}=(\mathcal{V}, \mathcal{E})$ be an undirected and connected graph defined by the set of nodes (agents) $\mathcal{V}$ and the set of edges $\mathcal{E} \subset \mathcal{V} \times \mathcal{V}$. Since $\mathcal{G}$ is undirected, we assume that both $(i, j)$ and $(j, i)$ refer to the same edge when it exists.

Let $X$ be a finite dimensional Hilbert space. For a closed convex set $C$, let $\delta_{C}(\cdot)$ be the indicator function defined as $\delta_{C}(x)=0$ if $x \in C$, and equals $\infty$ otherwise. For each edge $(i, j) \in \mathcal{E}$, let the hyperplane $H_{(i, j)} \subset X^{|\mathcal{V}|}$ be defined by

$$
H_{(i, j)}=\left\{\left(x_{1}, \ldots, x_{n}\right): x_{i}=x_{j}\right\} .
$$

We consider the following problem throughout the rest of this paper.

Problem 1.1. Let $(\mathcal{V}, \mathcal{E})$ be a connected graph. Suppose $H_{(i, j)}$ is defined as in (1.1) for all $(i, j) \in \mathcal{E}$, and let $f_{i}: X \rightarrow \overline{\mathbb{R}}$ (where $\overline{\mathbb{R}}:=\mathbb{R} \cup\{\infty\}$ throughout this paper) be closed convex functions for all $i \in \mathcal{V}$. Let $\mathbf{f}_{i}: X^{|\mathcal{V}|} \rightarrow \overline{\mathbb{R}}$ be defined by $\mathbf{f}_{i}(x)=f_{i}\left(x_{i}\right)$ (i.e., $\mathbf{f}_{i}$ depends only on $i$-th variable). The primal problem of interest is

$$
\min _{x \in X^{|\mathcal{V}|}} \sum_{(i, j) \in \mathcal{E}} \underbrace{\delta_{H_{(i, j)}}(x)}_{h_{(i, j)}(x)}+\sum_{i \in \mathcal{V}} \underbrace{\mathbf{f}_{i}(x)}_{h_{i}(x)} .
$$

For each $\alpha \in \mathcal{E} \cup \mathcal{V}$, the function $h_{\alpha}: X^{|\mathcal{V}|} \rightarrow \overline{\mathbb{R}}$ is as marked in (1.2). Since $(\mathcal{V}, \mathcal{E})$ is connected, the problem (1.2) is equivalent to

$$
\min _{x \in X} \sum_{i \in \mathcal{V}} f_{i}(x)
$$

but we write it in the form (1.2) to emphasize that the only vertex which has knowledge of the function $f_{i}(\cdot)$ is the vertex $i$.

\footnotetext{
* Submitted to the editors DATE.

Funding: Grant R-146-000-214-112 from the Faculty of Science, National University of Singapore

${ }^{\dagger}$ Department of Mathematics, National University of Singapore (matpchj@nus.edu.sg,http:// www.math.nus.edu.sg/ $\sim$ matpchj).
} 
1.1. Distributed algorithms for (1.2). We give a brief summary of distributed algorithms for minimizing (1.2). Some properties desirable for a distributed algorithm, especially when $|\mathcal{V}|$ is large, are as follows:

1. The algorithm is applicable to directed graphs, where only one way communication is allowed between two vertices connected by a directed edge.

2. The algorithm has deterministic convergence.

3. The algorithm is asynchronous. There is no need for a global clock, and each node can perform calculations at its own pace without being affected by other slower nodes.

4. The algorithm is distributed (i.e., in intermediate computations, each node only exchanges data with its neighbors) and decentralized (i.e., there is no central node connected to all other nodes to coordinate computations).

5. The algorithm allows for time-varying graphs.

We emphasize that the algorithm that we look at in this paper is only applicable to undirected graphs, and hence does not satisfy property (1). Nevertheless, we give a brief summary of the literature behind distributed algorithms for directed graphs in this paragraph. In the case where only one way communication is allowed between two vertices connected by a directed edge, the survey [30] records many algorithms derived from the subgradient algorithm for solving (1.2). If the edges in a network are directed, it appears that the subgradient method is the only reasonable method. The subgradient method requires diminishing step sizes for convergence in the general case, which affects its convergence rates. More details of recent developments are in [31]. A notable paper is [42]. The case of time-varying graphs was first studied in [32] and further extended in [33]. In time-varying graphs, the assumption needed for convergence is for the edge set $\mathcal{E}$ to vary over time. But if the edges are undirected, then alternative methods may be possible, and would usually be faster than subgradient methods. For strongly convex problems, linear convergence is possible. These algorithms appear to be synchronous, and require the functions involved to be smooth.

Two common methods for minimizing the sum of two convex functions are the ADMM and Peaceman-Rachford algorithms (with the Douglas Rachford algorithm a special case of the latter). The Peaceman-Rachford algorithm is an example of a splitting method, and it is well known that the ADMM is dual to the Douglas Rachford method [23]. In order to minimize the sum of more than two functions, the product space reformulation is a well-studied option. (See for example [11, Chapter 7].) Another strategy is [21, 16], which is a splitting method for the sum of more than two functions without using the product space reformulation. The latter development in [16] allows for lags in the collection of data for nodes where the computation time is greater, thus allowing for an asynchronous operation. Still, this algorithm requires a central controller, so it is different from the algorithms we consider in this paper.

We now look at asynchronous distributed algorithm with deterministic convergence (rather than probabilistic convergence). In some applications, the guarantees from deterministic convergence can outweigh other advantages of algorithms with randomized convergence. We mention that the paper [25] and the extension [3] are algorithms that give deterministic convergence for strongly convex problems that are primal in nature, so these algorithms cannot handle more than one constraint sets. The method in [2] may arguably be considered to have these properties. Other than that, we are not aware of a decentralized, asynchronous algorithm that has deterministic convergence for (1.2) and is not a subgradient method.

But a decentralized asynchronous probabilistic method derived from the ADMM is 
proposed in $[28,9]$. The key idea in the first paper is the introduction of a randomized Gauss-Seidel iterations of the so called Douglas-Rachford operator, and the second paper extends the first by incorporating the work of $[46,17]$. This concept was generalized in [39]. All the works just mentioned use monotone operator theory (see for example the textbook [5]). Such algorithms require computations in the nodes to follow specific probability distributions, so they do not seem immediately applicable to the setting of time-varying graphs in [32] mentioned earlier. Another randomized, distributed method for nonconvex functions is [13].

Another randomized distributed method most similar to what we discuss in this paper is that in [35]. We discuss this more in Subsection 1.2.

As mentioned in the survey [30], the primal problem (1.3) has dual

$$
\begin{gathered}
\max _{\left\{y_{i}\right\}_{i \in \mathcal{V} \subset X}}-\sum_{i \in \mathcal{V}} f_{i}^{*}\left(y_{i}\right) \\
\text { s.t. } \quad \sum_{i \in \mathcal{V}} y_{i}=0,
\end{gathered}
$$

which is also known as the resource allocation problem.

1.2. A special case of (1.2) through Dykstra's algorithm. When some of the functions in the primal problem of the form (1.3) are extended valued, it may be difficult to find a primal feasible point in the first place. We first look at the problem

$$
\min _{x \in X^{|\mathcal{V}|}} \frac{1}{2}\left\|x-x_{0}\right\|^{2}+\sum_{(i, j) \in \mathcal{E}} \delta_{H_{(i, j)}}(x)+\sum_{i \in \mathcal{V}} \mathbf{f}_{i}(x),
$$

where $\|x\|^{2}:=\langle x, x\rangle=\sum_{i=1}^{n}\left\langle x_{i}, x_{i}\right\rangle$ and $x_{0} \in X^{|\mathcal{V}|}$. This problem fits the framework of (1.2) since

$$
\frac{1}{2}\left\|x-x_{0}\right\|^{2}+\sum_{i \in \mathcal{V}} \mathbf{f}_{i}(x)=\sum_{i \in \mathcal{V}}\left[\mathbf{f}_{i}(x)+\frac{1}{2}\left\|x_{i}-\left[x_{0}\right]_{i}\right\|^{2}\right] .
$$

In the case when $\mathbf{f}_{i} \equiv 0$ for all $i \in \mathcal{V}$, the problem reduces to the average consensus problem $[10,19]$. The minimizer to $(1.5)$ is the vector $(\bar{x}, \ldots, \bar{x}) \in X^{|\mathcal{V}|}$, where $\bar{x}=\frac{1}{|\mathcal{V}|} \sum_{i \in \mathcal{V}}\left[x_{0}\right]_{i}$.

The (Fenchel) dual to (1.5) is

$$
\max _{z_{\alpha} \in X^{|\mathcal{V}|, \alpha \in \mathcal{E} \cup \mathcal{V}}} F\left(\left\{z_{\alpha}\right\}_{\alpha \in \mathcal{E} \cup \mathcal{V})}\right.
$$

where

$$
\begin{aligned}
& F\left(\left\{z_{\alpha}\right\}_{\alpha \in \mathcal{E} \cup \mathcal{V})}\right. \\
:= & -\frac{1}{2}\left\|x_{0}-\sum_{\alpha \in \mathcal{E} \cup \mathcal{V}} z_{\alpha}\right\|^{2}+\frac{1}{2}\left\|x_{0}\right\|^{2}-\sum_{(i, j) \in \mathcal{E}} \delta_{H_{(i, j)}}^{*}\left(z_{(i, j)}\right)-\sum_{i \in \mathcal{V}} \mathbf{f}_{i}^{*}\left(z_{i}\right) .
\end{aligned}
$$

In the case when $f_{i}(\cdot) \equiv \delta_{C_{i}}(\cdot)$ for some closed convex set $C_{i}$ for all $i$, Dykstra's algorithm finds the primal minimizer of the problem

$$
\min _{x \in X} \frac{1}{2}\left\|x-x_{0}\right\|^{2}+\sum_{i=1}^{n} f_{i}(x)
$$


where $x_{0} \in X$ (note that in (1.9), $x$ and $x_{0}$ lie in $X$ instead of $X^{|\mathcal{V}|}$ like in (1.5)) by maximizing the dual

$$
\max _{y \in X^{n}}-\frac{1}{2}\left\|x_{0}-\sum_{i=1}^{n} y_{i}\right\|-\sum_{i=1}^{n} f_{i}^{*}\left(y_{i}\right)+\frac{1}{2}\left\|x_{0}\right\|^{2}
$$

through block coordinate minimization. If each $f_{i}(\cdot)$ are allowed to be any closed convex function, it can now be seen that (1.8) is actually a special case of (1.10).

Dykstra's algorithm was first studied in [20] in the case where $f_{i}(\cdot) \equiv \delta_{C_{i}}(\cdot)$ and $C_{i}$ are closed convex sets for all $i$. The convergence of the primal iterates to the projection of $x_{0}$ onto $\cap_{i=1}^{n} C_{i}$ was proved in [12], and is sometimes called the BoyleDykstra theorem. Dykstra's algorithm was independently noted in [26] to be block coordinate minimization on the dual problem. The proof in [12] was adapted in [24] using duality. We remark that the Boyle-Dykstra theorem is remarkable because the convergence to the primal minimizer occurs even when there is no dual optimizer. (For example, look at [26, page 9$]$ where two circles in $\mathbb{R}^{2}$ intersect at only one point.) The case when sampling of the sets is noncyclic is addressed in [27] (among other things not directly relevant to this paper). As pointed out in [38], the Boyle-Dykstra theorem holds even if $f_{i}(\cdot)$ are closed convex functions instead of $\delta_{C_{i}}(\cdot)$. (We recently became aware that the dual ascent interpretation can be traced to $[14,15,1]$, but the connection to distributed optimization was not pointed out there.) For more on the background on Dykstra's algorithm, we refer to [5, 4, 18, 22]. Some recent work on Dykstra's algorithm include [43].

Dykstra's algorithm was extended to a distributed algorithm in [40], and they highlight the works $[2,29,41,36]$ on distributed optimization. The work in [40] is vastly different from how Dykstra's algorithm is studied in [12] and [24].

It turns out that [35] discusses a similar problem to (1.5). They generalize (1.5) by allowing the functions $x_{i} \mapsto \frac{1}{2}\left\|x_{i}-\left[x_{0}\right]_{i}\right\|^{2}$ to be any strongly convex function, and proceed to calculate that the dual has a similar form as (1.7) and (1.8). Their dual is still a sum of a smooth component and a separable component, which they solve with randomized dual proximal gradient. We discuss the differences between their paper and ours in Subsection 1.3.

1.3. Contributions of this paper. In this paper, we propose looking at the formulation (1.5) and show that Dykstra's algorithm applied to this formulation gives an algorithm with properties (2)-(5) in Subsection 1.1. As stated in the introduction, we are not aware of any other asynchronous distributed algorithm that has deterministic convergence other than $[25,3,2]$, though our assumption that the functions on each vertex has a strongly convex function with known modulus might be a bit strong.

We highlight the differences from [35]. The first difference is that we show that Dykstra's algorithm gives deterministic convergence (property (2)), whereas [35] pointed out probabilistic convergence. A naive application of Dykstra's algorithm to (1.5) would mean that all the edges in the graph have to be used in one cycle, which would not cover the setting of time-varying graphs as done in [32]. But we show that as long as the graph is (using the definition in [32]) uniformly connected, then convergence can be achieved. (See Remark 2.13.) Dykstra's splitting also gives these two desirable properties that were not noticed in [35]:

(6) The iterates of the algorithm converges to the primal minimizer even when a dual minimizer does not exist.

(7) Since Dykstra's splitting is a dual ascent algorithm, as many dual variables can be maximized at one time as possible. This is an advantage as sub- 
problems involving more dual variables lead to greedier, and possibly greater, increase of the dual objective value.

Next, in Section 3, we look at a decentralized dual ascent algorithm for (1.2) (which does not have the quadratic term) through the dual problem (1.4). Once again, the algorithm is asynchronous. In contrast to the adapted Dykstra's algorithm, we now optimize dual variables corresponding to a collection of vertices at a time. We show an example where convergence fails. The algorithm works on collections of vertices of the graph at a time, and is thus robust to lost communications in edges of the graph.

Lastly, in Section 4, we discuss ideas for an accelerated proximal gradient method on the dual (1.7). This algorithm runs on a global clock, and it does not work for time-varying graphs. (I.e., it does not satisfy properties (4)-(5)). But asynchronous greedy steps satisfying property (7) can be performed to speed up the increase of the dual objective value.

2. Convergence of distributed Dykstra's algorithm. In this section, we state our distributed Dykstra's algorithm, make some remarks that may be helpful in understanding the algorithm, and prove its convergence without constraint qualifications.

2.1. Statement of distributed Dykstra's algorithm. Let $D \subset X^{|\mathcal{V}|}$ be the diagonal set defined by

$$
D:=\left\{x \in X^{|\mathcal{V}|}: x_{1}=x_{2}=\cdots=x_{|\mathcal{V}|}\right\} .
$$

With the definition of $H_{(i, j)}$ in $(1.1)$ and $\mathcal{G}=(\mathcal{V}, \mathcal{E})$ being a connected graph, it is obvious that

$$
\bigcap_{(i, j) \in \mathcal{E}} H_{(i, j)}=D \text { and } \sum_{(i, j) \in \mathcal{E}} H_{(i, j)}^{\perp}=D^{\perp}=\left\{z \in X^{|\mathcal{V}|}: \sum_{i \in \mathcal{V}} z_{i}=0\right\} .
$$

Proposition 2.1. Suppose $\mathcal{G}=(\mathcal{V}, \mathcal{E})$ is a connected graph. Let $H_{(i, j)}$ be the set (1.1) (defining the linear constraints relating the connection between nodes $i$ and $j$ ). Let $\mathcal{E}^{\prime}$ be a subset of $\mathcal{E}$. The following conditions are equivalent:

1. $\cap_{(i, j) \in \mathcal{E}^{\prime}} H_{(i, j)}=D$

2. $\sum_{(i, j) \in \mathcal{E}^{\prime}} H_{(i, j)}^{\perp}=D^{\perp}$.

3. The graph $\mathcal{G}^{\prime}=\left(\mathcal{V}, \mathcal{E}^{\prime}\right)$ is connected.

Proof. The equivalence between (1) and (3) is easy, and the equivalence between (1) and (2) is simple linear algebra.

Definition 2.2. We say that $\mathcal{E}^{\prime}$ connects $\mathcal{V}$ if any of the equivalent properties in Proposition 2.1 is satisfied.

We prove a lemma.

Lemma 2.3. (Expressing $v$ as a sum) Suppose $X$ is a finite dimensional Hilbert space. There is a $C_{1}>0$ such that for all $v \in D^{\perp}$ and $\mathcal{E}^{\prime} \subset \mathcal{E}$ such that $\mathcal{E}^{\prime}$ connects $\mathcal{V}$, we can find $z_{(i, j)} \in H_{(i, j)}^{\perp}$ for all $(i, j) \in \mathcal{E}^{\prime}$ such that $\sum_{(i, j) \in \mathcal{E}^{\prime}} z_{(i, j)}=v$ and $\left\|z_{(i, j)}\right\| \leq C_{1}\|v\|$ for all $(i, j) \in \mathcal{E}^{\prime}$.

Proof. This is elementary, so we only give an outline. Fix an $\mathcal{E}^{\prime}$. We can choose $\tilde{H}_{(i, j)} \subset H_{(i, j)}^{\perp}$ so that $\sum_{(i, j) \in \mathcal{E}^{\prime}} H_{(i, j)}^{\perp}=D^{\perp}$ is a direct sum of $\left\{\tilde{H}_{(i, j)}\right\}_{(i, j) \in \mathcal{E}^{\prime}}$. So $v$ can be written uniquely as the $\operatorname{sum} v=\sum_{(i, j) \in \mathcal{E}^{\prime}} z_{(i, j)}$, where $z_{(i, j)} \in \tilde{H}_{(i, j)}$. The mapping from $v$ to each $z_{(i, j)} \in \tilde{H}_{(i, j)}$ is linear, and this linear map has a norm 
bounded by some $C_{(i, j), \mathcal{E}^{\prime}}$. Letting $C_{1}$ be the maximum of these $C_{(i, j), \mathcal{E}^{\prime}}$ gives us our conclusion.

We present our distributed Dykstra's algorithm in Algorithm 2.1.

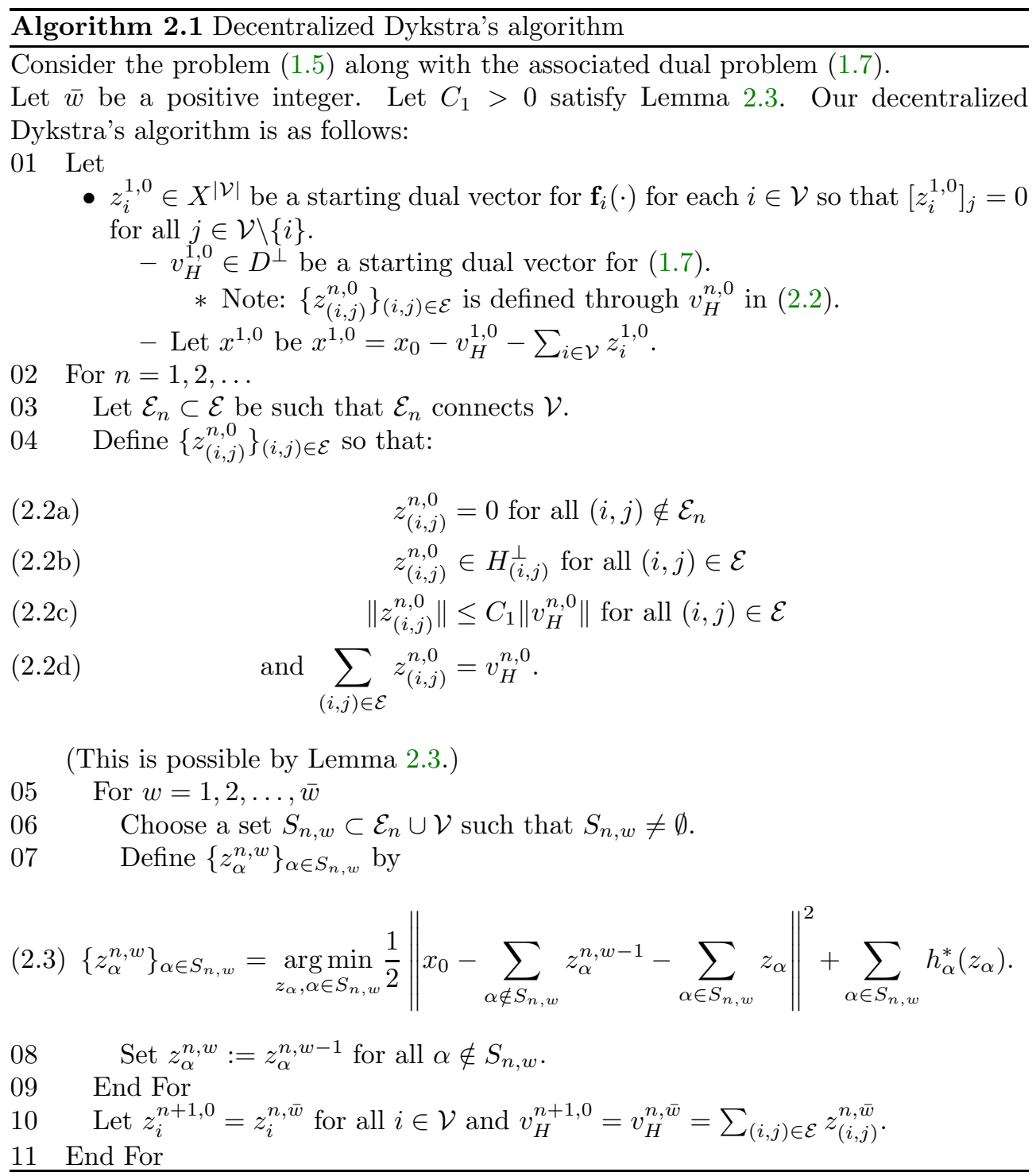

Remark 2.4. (Intuition behind Algorithm 2.1) We now provide some intuition behind Algorithm 2.1. The classical Dykstra splitting approach is the block coordinate maximization of the dual problem (1.7)-(1.8). This is reflected in lines $6-8$ of Algorithm 2.1. In order for Algorithm 2.1 to handle time-varying graphs, we choose $\mathcal{E}_{n} \subset \mathcal{E}$ in line 3 so that $\mathcal{E}_{n}$ connects $\mathcal{V}$, and the problem

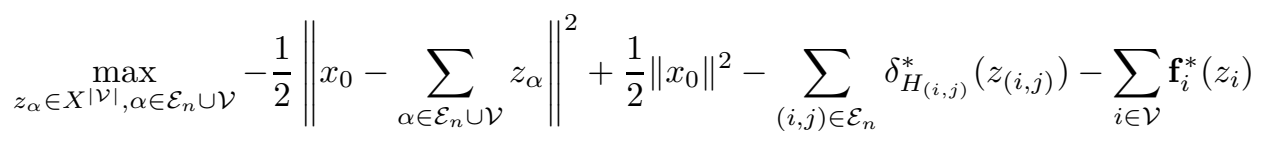


(note the $\mathcal{E}_{n}$ in the above formula) would have the same optimal objective value as (1.7)-(1.8) since the corresponding primal problems are equivalent and strong duality holds. The subset $\mathcal{E}_{n} \subset \mathcal{E}$ chosen in line 3 may be such that $z_{(i, j)} \neq 0$, but $(i, j) \notin \mathcal{E}_{n}$. So we perform line 4 so that (2.2) holds, which implies that $z_{(i, j)}=0$ for all $(i, j) \notin \mathcal{E}_{n}$, while perserving $v_{H}^{n, \bar{w}}=v_{H}^{n+1,0}$ (see (2.4) later). As we shall see in Remark 2.10 later, the reassignment of $\left\{z_{(i, j)}\right\}_{(i, j) \in \mathcal{E}}$ in line 4 is necessary for further analysis, but may be ignored in implementing the algorithm. Algorithm 2.2 then shows an equivalent formulation of Algorithm 2.1 where one only keeps track of $x^{n, w}$ and $\left\{z_{i}^{n, w}\right\}_{i \in \mathcal{V}}$. If $f_{i}(\cdot) \equiv 0$, then $z_{i}^{n, w}$ is always 0 for all $i \in \mathcal{V}$, so Algorithm 2.2 reduces to the averaged consensus algorithm [10, 19].

Remark 2.5. (Choice of $S_{n, w}$ ) The choice of $S_{n, w}$ allows for a flexibility in how large one wants the subproblem (2.3) to be. It is easy to see that a small $S_{n, w}$ allows for the subproblems to be small and easy to solve. The larger the size of $S_{n, w}$, the harder the subproblem, but greater increase in the dual objective value is expected. An issue of choosing large $\left|S_{n, w}\right|$ is that we need an extra coordination between the nodes in $V_{n, w}^{\prime}$. When $S_{n, w} \subset \mathcal{V}$ and $\left|S_{n, w}\right|=1$, there is no coordination needed. Similarly, when $S_{n, w} \subset \mathcal{E}$ and $\left|S_{n, w}\right|=1$, only two nodes need to coordinate with each other, which is okay for an undirected graph. An implementer can, for example, choose a star-like subgraph (i.e., there is a central node in the subgraph connecting to all others) and apply an algorithm suitable for problems with a centralized node. Since the dual objective value acts as a Lyapunov function, one could choose $S_{n, w}$ to be as large as one can reasonably solve to increase the dual objective value as much as one can. This increase in the dual objective value can be greater if large subproblems are solved partially compared to small subproblems solved fully.

To simplify calculations, we let $v_{A}, v_{H}$ and $x$ be denoted by

$$
\begin{aligned}
v_{H} & =\sum_{(i, j) \in \mathcal{E}} z_{(i, j)} \\
v_{A} & =v_{H}+\sum_{i \in \mathcal{V}} z_{i} \\
x & =x_{0}-v_{A} .
\end{aligned}
$$

Intuitively, $v_{H}$ describes the sum of the dual variables due to $H_{(i, j)}$ for all $(i, j) \in \mathcal{E}$, $v_{A}$ is the sum of all dual variables, and $x$ is the estimate of the primal variable.

The following inequality describes the duality gap between (1.5) and (1.7).

$$
\begin{aligned}
& \frac{1}{2}\left\|x_{0}-x\right\|^{2}+\sum_{\alpha \in \mathcal{E} \cup \mathcal{V}} h_{\alpha}(x)-F\left(\left\{z_{\alpha}\right\}_{\alpha \in \mathcal{E} \cup \mathcal{V})}\right) \\
& \stackrel{(1.8)}{=} \quad \frac{1}{2}\left\|x_{0}-x\right\|^{2}+\sum_{\alpha \in \mathcal{E} \cup \mathcal{V}}\left[h_{\alpha}(x)+h_{\alpha}^{*}\left(z_{\alpha}\right)\right] \\
& -\left\langle x_{0}, \sum_{\alpha \in \mathcal{E} \cup \mathcal{V}} z_{\alpha}\right\rangle+\frac{1}{2}\left\|\sum_{\alpha \in \mathcal{E} \cup \mathcal{V}} z_{\alpha}\right\|^{2} \\
& \stackrel{\text { Fenchel duality }}{\geq} \frac{1}{2}\left\|x_{0}-x\right\|^{2}+\left\langle x, \sum_{\alpha \in \mathcal{E} \cup \mathcal{V}} z_{\alpha}\right\rangle-\left\langle x_{0}, \sum_{\alpha \in \mathcal{E} \cup \mathcal{V}} z_{\alpha}\right\rangle+\frac{1}{2}\left\|\sum_{\alpha \in \mathcal{E} \cup \mathcal{V}} z_{\alpha}\right\|^{2} \\
& =\quad \frac{1}{2}\left\|x_{0}-x-\sum_{\alpha \in \mathcal{E} \cup \mathcal{V}} z_{\alpha}\right\|^{2} \geq 0 .
\end{aligned}
$$

Claim 2.6. In Algorithm 2.1, for all $\alpha \in S_{n, w}$, we have 
(a) $-x^{n, w}+\partial h_{\alpha}^{*}\left(z_{\alpha}^{n, w}\right) \ni 0$,

(b) $-z_{\alpha}^{n, w}+\partial h_{\alpha}\left(x^{n, w}\right) \ni 0$, and

(c) $h_{\alpha}\left(x^{n, w}\right)+h_{\alpha}^{*}\left(z_{\alpha}^{n, w}\right)=\left\langle x^{n, w}, z_{\alpha}^{n, w}\right\rangle$.

Proof. By taking the optimality conditions in (2.3) with respect to $z_{\alpha}$ for $\alpha \in S_{n, w}$ and making use of (2.4) to get $x^{n, w}=x_{0}-\sum_{\alpha \in \mathcal{V} \cup \mathcal{E}} z_{\alpha}^{n, w}$, we deduce (a). The equivalence of (a), (b) and (c) is standard.

Even though Algorithm 2.1 is described so that each node $i \in \mathcal{V}$ and edge $(i, j) \in \mathcal{E}$ contains a variable $z_{\alpha} \in X^{|\mathcal{V}|}$, the size of the variable $z_{\alpha}$ that needs to be stored in each node and edge is small due to sparsity.

Proposition 2.7. (Sparsity of $z_{\alpha}$ ) We have $\left[z_{i}^{n, w}\right]_{j}=0$ for all $j \in \mathcal{V} \backslash\{i\}, n \geq 1$ and $w \in\{0,1, \ldots, \bar{w}\}$. Similarly, $\left[z_{(i, j)}^{n, w}\right]_{k}=0$ for all $k \in \mathcal{V} \backslash\{i, j\}, n \geq 1$ and $w \in\{0,1, \ldots, \bar{w}\}$.

Proof. The result for $z_{i}^{n, w}$ holds for $n=1$ and $w=0$. Claim 2.6(b) shows that $z_{i}^{n, w} \in \partial \mathbf{f}_{i}\left(x^{n, w}\right)$ for all $i \in S_{n, w}$. Note that since $\left[\mathbf{f}_{i}(x)\right]_{j}=0$ for all $j \in \mathcal{V} \backslash\{i\}$, $\left[\partial \mathbf{f}_{i}(x)\right]_{j}=0$ for all $j \in \mathcal{V} \backslash\{i\}$, which easily gives what we need.

For all $(i, j) \in \mathcal{E}$ and $n \geq 1$, the line (2.2b) implies that $z_{(i, j)}^{n, 0} \in H_{(i, j)}^{\perp}$, and Claim 2.6(b) implies that $z_{(i, j)}^{n, w} \in H_{(i, j)}^{\perp}$ for all $w \in\{1, \ldots, \bar{w}\}$. This implies the result at hand for $z_{(i, j)}^{n, w}$.

Dykstra's algorithm is traditionally written in terms of solving for the primal variable $x$. For completeness, we show the equivalence between (2.3) and the primal minimization problem. The proof is easily extended from [38, Proposition 2.4] (The duality between (2.3) and (2.6) can also be obtained by Fenchel duality.)

Proposition 2.8. (On solving (2.3)) If a minimizer $\left\{z_{\alpha}^{n, w}\right\}_{\alpha \in S_{n, w}}$ for (2.3) exists, then the $x^{n, w}$ in (2.4c) satisfies

$$
x^{n, w}=\underset{x \in X^{|\mathcal{V}|}}{\arg \min } \sum_{\alpha \in S_{n, w}} h_{\alpha}(x)+\frac{1}{2}\left\|x-\left(x_{0}-\sum_{\alpha \notin S_{n, w}} z_{\alpha}^{n, w}\right)\right\|^{2} .
$$

Conversely, if $x^{n, w}$ solves (2.6) with the dual variables $\left\{\tilde{z}_{\alpha}^{n, w}\right\}_{\alpha \in S_{n, w}}$ satisfying

$$
\tilde{z}_{\alpha}^{n, w} \in \partial h_{\alpha}\left(x^{n, w}\right) \text { and } x^{n, w}-x_{0}+\sum_{\alpha \notin S_{n, w}} z_{\alpha}^{n, w}+\sum_{\alpha \in S_{n, w}} \tilde{z}_{\alpha}^{n, w}=0,
$$

then $\left\{\tilde{z}_{\alpha}^{n, w}\right\}_{\alpha \in S_{n, w}}$ solves (2.3).

2.2. Examples of $S_{n, w}$. In this subsection, we elaborate on how to solve (2.6), and show that Algorithm 2.1 is an extension of the average consensus algorithm.

For an $S_{n, w}$ such that $S_{n, w} \cap \mathcal{E} \neq \emptyset$, define $\mathcal{V}_{n, w}^{\prime}$ by

$$
\mathcal{V}_{n, w}^{\prime}=\left\{\text { all vertices that are endpoints of some edge in } S_{n, w} \cap \mathcal{E}\right\} .
$$

Suppose $S_{n, w} \cap \mathcal{E}$ is such that the subgraph $\left(\mathcal{V}_{n, w}^{\prime}, S_{n, w} \cap \mathcal{E}\right)$ is a connected graph with no cycles, and $S_{n, w} \cap \mathcal{V} \subset \mathcal{V}_{n, w}^{\prime}$. Let $\tilde{y} \in X^{|\mathcal{V}|}$ be defined by

$$
\tilde{y}:=x_{0}-\sum_{\alpha \notin S_{n, w}} z_{\alpha}^{n, w} \stackrel{\text { line }}{=} x_{0}-\sum_{\alpha \notin S_{n, w}} z_{\alpha}^{n, w-1} \stackrel{(2.4)}{=} x^{n, w-1}+\sum_{\alpha \in S_{n, w}} z_{\alpha}^{n, w-1} .
$$


Then the primal minimization problem (2.6) becomes

$$
\begin{gathered}
x^{n, w}=\underset{x \in X^{|\mathcal{V}|}}{\arg \min } \sum_{i \in S_{n, w} \cap \mathcal{V}} h_{i}(x)+\sum_{(i, j) \in S_{n, w} \cap \mathcal{E}} h_{(i, j)}(x) \\
+\frac{1}{2}\left\|x-\left(x_{0}-\sum_{\alpha \notin S_{n, w}} z_{\alpha}^{n, w}\right)\right\|^{2} \\
\stackrel{(2.9)}{=} \underset{x \in X^{|\mathcal{V}|}}{\arg \min } \sum_{i \in S_{n, w} \cap \mathcal{V}} h_{i}(x)+\sum_{(i, j) \in S_{n, w} \cap \mathcal{E}} h_{(i, j)}(x)+\frac{1}{2}\|x-\tilde{y}\|^{2} .
\end{gathered}
$$

Recall that $h_{i}: X^{|\mathcal{V}|} \rightarrow \mathbb{R}$ is a function whose output depends only on the $i$-th coordinate, where $i \in \mathcal{V}$. If $x^{n, w}$ were to solve $(2.10)$, then $h_{(i, j)}\left(x^{n, w}\right)=\delta_{H_{(i, j)}}\left(x^{n, w}\right)$ is finite for all $(i, j) \in S_{n, w} \cap \mathcal{E}$, which shows that $x^{n, w} \in H_{(i, j)}$ for all $(i, j) \in S_{n, w} \cap \mathcal{E}$. This in turn means that all the components of $x^{n, w}$ indexed by $\mathcal{V}_{n, w}^{\prime}$ would need to have the same value. So the problem (2.10) can be reduced to one which optimizes over a variable in $X$ (instead of $X^{|\mathcal{V}|}$ ), which, for all $i^{\prime} \in \mathcal{V}_{n, w}^{\prime}$, takes the form

$$
\begin{aligned}
x_{i^{\prime}}^{n, w} \stackrel{(2.10)}{=} \underset{x \in X}{\arg \min } \sum_{i \in S_{n, w} \cap \mathcal{V}} f_{i}(x)+\frac{1}{2} \sum_{i \in \mathcal{V}_{n, w}^{\prime}}\left\|x-\tilde{y}_{i}\right\|^{2} \\
=\underset{x \in X}{\arg \min } \sum_{i \in S_{n, w} \cap \mathcal{V}} f_{i}(x)+\frac{\left|\mathcal{V}_{n, w}^{\prime}\right|}{2}\left\|x-\frac{1}{\left|\mathcal{V}_{n, w}^{\prime}\right|} \sum_{i \in \mathcal{V}_{n, w}^{\prime}} \tilde{y}_{i}\right\|^{2}
\end{aligned}
$$

where $f_{i}: X \rightarrow \overline{\mathbb{R}}$ is defined as in Problem 1.1. The iterate $x^{n, w} \in X^{|\mathcal{V}|}$ can be expressed in terms of $x^{n, w-1}$ via

$$
x_{i^{\prime}}^{n, w}= \begin{cases}x_{i^{\prime}}^{n, w-1} & \text { if } i^{\prime} \notin \mathcal{V}_{n, w}^{\prime} \\ \text { The formula in (2.11) } & \text { if } i^{\prime} \in \mathcal{V}_{n, w}^{\prime} .\end{cases}
$$

Remark 2.9. (The case $S_{n, w} \cap \mathcal{V}=\emptyset$ ) A notable case is when $S_{n, w} \cap \mathcal{V}=\emptyset$ and $\left|S_{n, w} \cap \mathcal{E}\right|=1$. Let $(i, j)$ be the element in $S_{n, w} \cap \mathcal{E}$. Then one can calculate from (2.12) that $x_{i}^{n, w}=x_{j}^{n, w}=\frac{1}{2}\left(x_{i}^{n, w-1}+x_{j}^{n, w-1}\right)$, and the other $|\mathcal{V}|$ components of $x^{n, w}$ remain unchanged from $x^{n, w-1}$. If $f_{i}(\cdot) \equiv 0$ for all $i \in \mathcal{V}$ and the edges are chosen over the graph $(\mathcal{V}, \mathcal{E})$, we reduce to the case of averaged consensus studied in $[10,19]$.

2.3. Simplification of Algorithm 2.1 and further remarks. We first remark that there is no need to track $\left\{z_{(i, j)}^{n, w-1}\right\}_{(i, j) \in \mathcal{E}}$ throughout the algorithm, and we only need to keep track of $\left\{z_{i}^{n, w-1}\right\}_{i \in \mathcal{V}}$ and $x^{n, w-1}$. We make a few more remarks about Algorithm 2.1.

Remark 2.10. (Irrelevance of $\left.z_{(i, j)}^{n, w-1}\right)$ A first observation of the dual objective function is that as long as $z_{(i, j)} \in H_{(i, j)}^{\perp}$, we have $\delta_{H_{(i, j)}}^{*}\left(z_{(i, j)}\right)=\delta_{H_{(i, j)}^{\perp}}\left(z_{(i, j)}\right)=0$. Since

$$
-\frac{1}{2}\left\|x_{0}-\sum_{\alpha \in \mathcal{E} \cup \mathcal{V}} z_{\alpha}^{n, w}\right\|^{2} \stackrel{(2.4 a)}{=}-\frac{1}{2}\left\|x_{0}-v_{H}^{n, w}-\sum_{\alpha \in \mathcal{V}} z_{\alpha}^{n, w}\right\|^{2}
$$

the dual objective function (1.8) thus does not depend directly on each $\left\{z_{(i, j)}\right\}_{(i, j) \in \mathcal{E}}$, but rather through the sum $v_{H}:=\sum_{(i, j) \in \mathcal{E}} z_{(i, j)}$ that appears in the quadratic term in (2.3). Next, in calculating $\frac{1}{\mathcal{V}_{n, w}^{\prime}} \sum_{i \in \mathcal{V}_{n, w}^{\prime}} \tilde{y}_{i}$ in (2.11), we note that since $z_{(i, j)}^{n, w-1} \in$ 
$H_{(i, j)}^{\perp} \subset D^{\perp}, \sum_{i^{\prime} \in \mathcal{V}}\left[z_{(i, j)}^{n, w-1}\right]_{i^{\prime}} \stackrel{(2.1)}{=} 0$. Also, by Proposition 2.7, if $\alpha \in S_{n, w} \cap \mathcal{E}$, then $\left[z_{\alpha}^{n, w-1}\right]_{i}=0$ if $i \in \mathcal{V} \backslash \mathcal{V}_{n, w}^{\prime}$. This means that

$$
\begin{aligned}
& \sum_{i \in \mathcal{V}_{n, w}^{\prime}} \tilde{y}_{i} \stackrel{(2.9)}{=} \sum_{i \in \mathcal{V}_{n, w}^{\prime}}\left[x^{n, w-1}+\sum_{\alpha \in S_{n, w}} z_{\alpha}^{n, w-1}\right]_{i} \\
= & \sum_{i \in \mathcal{V}_{n, w}^{\prime}} x_{i}^{n, w-1}+\sum_{i \in \mathcal{V}_{n, w}^{\prime}} \sum_{\alpha \in S_{n, w} \cap \mathcal{V}}\left[z_{\alpha}^{n, w-1}\right]_{i}+\sum_{i \in \mathcal{V}_{n, w}^{\prime}} \sum_{\alpha \in S_{n, w} \cap \mathcal{E}}\left[z_{\alpha}^{n, w-1}\right]_{i} \\
= & \sum_{i \in \mathcal{V}_{n, w}^{\prime}} x_{i}^{n, w-1}+\sum_{i \in \mathcal{V}_{n, w}^{\prime}} \sum_{\alpha \in S_{n, w} \cap \mathcal{V}}\left[z_{\alpha}^{n, w-1}\right]_{i}+\sum_{i \in \mathcal{V}} \sum_{\alpha \in S_{n, w} \cap \mathcal{E}}\left[z_{\alpha}^{n, w-1}\right]_{i} \\
\stackrel{(2.1)}{=} & \sum_{i \in \mathcal{V}_{n, w}^{\prime}} x_{i}^{n, w-1}+\sum_{i \in \mathcal{V}_{n, w}^{\prime}} \sum_{\alpha \in S_{n, w} \cap \mathcal{V}}\left[z_{\alpha}^{n, w-1}\right]_{i} .
\end{aligned}
$$

So one only needs to keep track of $x^{n, w}$ and $\left\{z_{i}^{n, w}\right\}_{i \in \mathcal{V}}$ in Algorithm 2.1, and there

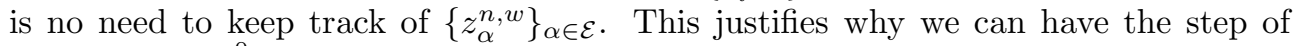
reassigning $z_{(i, j)}^{n, 0}$ in line 4, and Algorithm 2.1 could have been stated in terms of $v_{H}$ only, and not $\left\{z_{(i, j)}\right\}_{(i, j) \in \mathcal{E}}$. The reason why we need to introduce the variables $\left\{z_{(i, j)}\right\}_{(i, j) \in \mathcal{E}}$ is so that the analysis in (2.24) can be carried through.

In view of Remark 2.10, Algorithm 2.1 can thus be simplified to Algorithm 2.2 without the terms $\left\{z_{(i, j)}\right\}_{(i, j) \in \mathcal{E}}$. Furthermore, if $f_{i}(\cdot) \equiv 0$ for all $i \in V$, the variables $z_{i}^{n, w}$ would always be zero, and Algorithm 2.2 reduces to the well known averaged consensus problem [10, 19].

Remark 2.11. (Distributed asynchronous computation) Proposition 2.7 shows that the storage requirement for each vertex and edge is small. Suppose $S_{n, w}$ and $S_{n, w+1}$ are such that $S_{n, w} \cap \mathcal{V} \subset \mathcal{V}_{n, w}^{\prime}, S_{n, w+1} \cap \mathcal{V} \subset \mathcal{V}_{n, w+1}^{\prime}$ and $\mathcal{V}_{n, w}^{\prime} \cap \mathcal{V}_{n, w+1}^{\prime}=\emptyset$. Then the computations in for the iterations $(n, w)$ and $(n, w+1)$ can be conducted in parallel. This is because calculations for $S_{n, w}$ in (2.3) only uses and affects the coordinates of $\left\{z_{\alpha}\right\}_{\alpha \in \mathcal{E} \cup \mathcal{V}}$ indexed by $\mathcal{V}_{n, w}^{\prime} \subset \mathcal{V}$ and the similar thing goes for $S_{n, w+1}$. This idea can be naturally extended to the case of $S_{n, w}, S_{n, w+1}, \ldots, S_{n, w+j}$ for any $j \geq 1$ to allows for distributed asynchronous computation.

Remark 2.12. (Scalability) Algorithm 2.1 allows for the size of the sets $S_{n, w}$ to be arbitrarily large so that there would be a greedier increase in the dual objective value. One would then expect faster convergence with larger sizes of $S_{n, w}$. Even though for this paper, we only cover the case where $\left|S_{n, w} \cap \mathcal{V}\right| \leq 1$, the case where $\left|S_{n, w} \cap \mathcal{V}\right|>1$ can be analyzed using the techniques in [38], where we split vertices in $\mathcal{V}$ according to whether $\operatorname{dom}\left(f_{i}\right)=X, f_{i}(\cdot)$ is an indicator function of a closed convex set, or $f_{i}(\cdot)$ is a general closed convex function.

Remark 2.13. (Time-varying graphs) Note that in line 5 of Algorithm 2.1, we only need to choose $\mathcal{E}_{n} \subset \mathcal{E}$ so that $\mathcal{E}_{n}$ connects $\mathcal{V}$. As long as $\mathcal{E}_{n}=\left[\cup_{w=1}^{\bar{w}} S_{n, w}\right] \cap \mathcal{E}$, the convergence result in Theorem 2.14 holds. So as long as enough edges are chosen in each cycle to connect the graph, Algorithm 2.1 would converge. In [32], they used the term uniformly strongly connectedness or $B$-strongly connectedness for time-varying directed graphs. Our assumption is equivalent to how $B$-connectedness would have been defined for undirected graphs. 


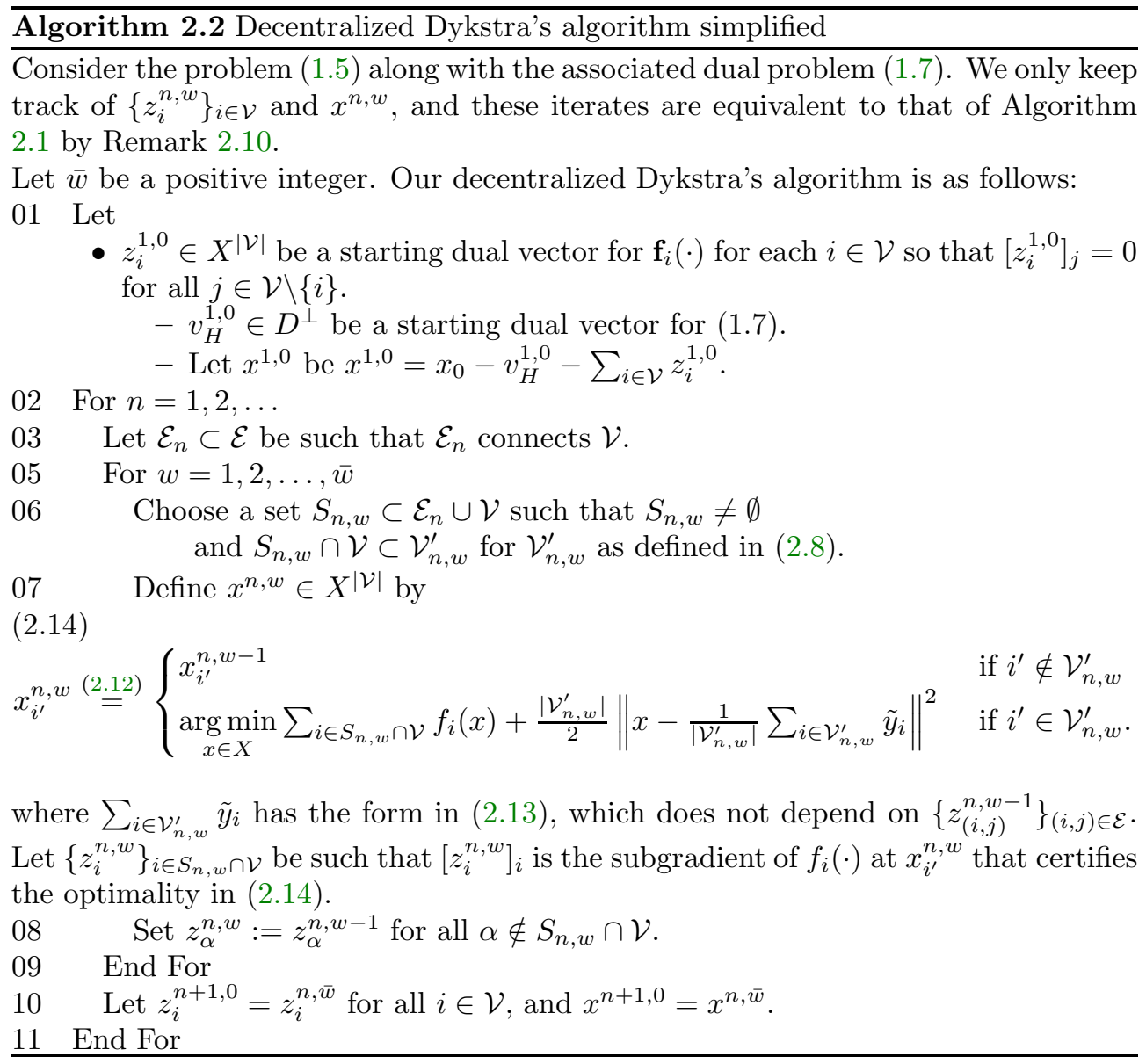

2.4. Convergence of Algorithm 2.1. We state some notation necessary for further discussions. For any $\alpha \in \mathcal{E} \cup \mathcal{V}$ and $n \in\{1,2, \ldots\}$, let $p(n, \alpha)$ be

$$
p(n, \alpha)=\max \left\{m: m \leq \bar{w}, \alpha \in S_{n, m}\right\} .
$$

In other words, $p(n, \alpha)$ is the index $m$ such that $\alpha \in S_{n, m}$ but $\alpha \notin S_{n, k}$ for all $k \in\{m+1, \ldots, \bar{w}\}$. It follows from line 8 in Algorithm 2.1 that

$$
z_{\alpha}^{n, p(n, \alpha)}=z_{\alpha}^{n, p(n, \alpha)+1}=\cdots=z_{\alpha}^{n, \bar{w}} .
$$

Moreover, $(i, j) \notin \mathcal{E}_{n}$ implies $(i, j) \notin S_{n, w}$ for all $w \in\{1, \ldots, \bar{w}\}$, so

$$
0 \stackrel{(2.2 \mathrm{a})}{=} z_{(i, j)}^{n, 0}=z_{(i, j)}^{n, 1}=\cdots=z_{(i, j)}^{n, \bar{w}} \text { for all }(i, j) \notin \mathcal{E}_{n} .
$$

We have the following theorem on the convergence of Algorithm 2.1.

TheOREm 2.14. (Convergence to primal minimizer) Consider Algorithm 2.1. Assume that for all $n \geq 1, \mathcal{E}_{n}=\left[\cup_{w=1}^{\bar{w}} S_{n, w}\right] \cap \mathcal{E}$, and $\left[\cup_{w=1}^{\bar{w}} S_{n, w}\right] \supset \mathcal{V}$. Assume that there are constants $A$ and $B$ such that

$$
\sum_{\alpha \in \mathcal{E} \cup \mathcal{V}}\left\|z_{\alpha}^{n, \bar{w}}\right\| \leq A \sqrt{n}+B \text { for all } n \geq 0 .
$$


For the sequence $\left\{z_{\alpha}^{n, w}\right\}_{\substack{1 \leq n<\infty \\ 0<w<w}} \subset X^{|\mathcal{V}|}$ for each $\alpha \in \mathcal{E} \cup \mathcal{V}$ generated by Algorithm 2.1 and the sequences $\left\{v_{H}^{n, w}\right\}_{\substack{1 \leq n<\infty \\ 0 \leq w \leq w}} \subset X^{|\mathcal{V}|}$ and $\left\{v_{A}^{n, w}\right\}_{\substack{1 \leq n<\infty \\ 0 \leq w \leq w}}^{\substack{0 \leq w \leq w \\ 0}} \subset X^{|\mathcal{V}|}$ thus derived, we have:

(i) The sum $\sum_{n=1}^{\infty} \sum_{w=1}^{\bar{w}}\left\|v_{A}^{n, w}-v_{A}^{n, w-1}\right\|^{2}$ is finite and $\left\{F\left(\left\{z_{\alpha}^{n, \bar{w}}\right\}_{\alpha \in \mathcal{E} \cup \mathcal{V})\}_{n=1}^{\infty}}\right.\right.$ is nondecreasing.

(ii) There is a constant $C$ such that $\left\|v_{A}^{n, w}\right\|^{2} \leq C$ for all $n \in \mathbb{N}$ and $w \in$ $\{1, \ldots, \bar{w}\}$.

(iii) There exists a subsequence $\left\{v_{A}^{n_{k}, \bar{w}}\right\}_{k=1}^{\infty}$ of $\left\{v_{A}^{n, \bar{w}}\right\}_{n=1}^{\infty}$ which converges to some $v_{A}^{*} \in X^{|\mathcal{V}|}$ and that

$$
\lim _{k \rightarrow \infty}\left\langle v_{A}^{n_{k}, \bar{w}}-v_{A}^{n_{k}, p\left(n_{k}, \alpha\right)}, z_{\alpha}^{n_{k}, \bar{w}}\right\rangle=0 \text { for all } \alpha \in \mathcal{E} \cup \mathcal{V} .
$$

(iv) For the $v_{A}^{*}$ in (iii), $x_{0}-v_{A}^{*}$ is the minimizer of the primal problem $(P)$ and we have $\lim _{k \rightarrow \infty} F\left(\left\{z_{\alpha}^{n_{k}, w}\right\}_{\alpha \in \mathcal{E} \cup \mathcal{V})}\right)=\frac{1}{2}\left\|v_{A}^{*}\right\|^{2}+h\left(x_{0}-v_{A}^{*}\right)$, where $h(\cdot)=$ $\sum_{\alpha \in \mathcal{E} \cup \mathcal{V}} h_{\alpha}(\cdot)$.

The properties (i) to (iv) in turn imply that $\lim _{n \rightarrow \infty} x^{n, \bar{w}}$ exists and equals $x_{0}-v_{A}^{*}$, which is the primal minimizer of (1.5).

Proof. We first show that (i) to (iv) implies the final assertion. For all $n \in \mathbb{N}$ we have, from weak duality,

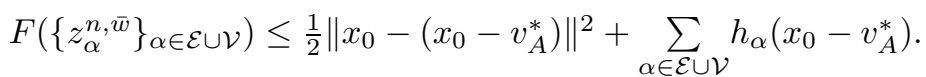

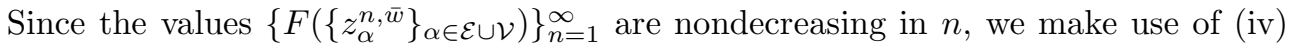
to get

$$
\lim _{n \rightarrow \infty} F\left(\left\{z_{\alpha}^{n, \bar{w}}\right\}_{\alpha \in \mathcal{E} \cup \mathcal{V}}\right)=\frac{1}{2}\left\|x_{0}-\left(x_{0}-v_{A}^{*}\right)\right\|^{2}+\sum_{\alpha \in \mathcal{E} \cup \mathcal{V}} h_{\alpha}\left(x_{0}-v_{A}^{*}\right),
$$

Hence $x_{0}-v_{A}^{*}=\arg \min _{x} h(x)+\frac{1}{2}\left\|x-x_{0}\right\|^{2}$, and (substituting $x=x_{0}-v_{A}^{*}$ in (2.5))

$$
\begin{array}{cl} 
& \frac{1}{2}\left\|x_{0}-\left(x_{0}-v_{A}^{*}\right)\right\|^{2}+h\left(x_{0}-v_{A}^{*}\right)-F\left(\left\{z_{\alpha}^{n, \bar{w}}\right\}_{\alpha \in \mathcal{E} \cup \mathcal{V})}\right. \\
\stackrel{(2.5),(2.4 \mathrm{a}),(2.4 \mathrm{~b})}{\geq} & \frac{1}{2}\left\|x_{0}-\left(x_{0}-v_{A}^{*}\right)-v_{A}^{n, \bar{w}}\right\|^{2} \\
\stackrel{(2.4 \mathrm{c})}{=} & \frac{1}{2}\left\|x^{n, \bar{w}}-\left(x_{0}-v_{A}^{*}\right)\right\|^{2} .
\end{array}
$$

Hence $\lim _{n \rightarrow \infty} x^{n, \bar{w}}$ is the minimizer in $(\mathrm{P})$.

It remains to prove assertions (i) to (iv).

Proof of (i): From the fact that $\left\{z_{\alpha}^{n, w}\right\}_{\alpha \in S_{n, w}}$ minimize (2.3) (which includes the quadratic regularizer) we have

$$
F\left(\{ z _ { \alpha } ^ { n , w - 1 } \} _ { \alpha \in \mathcal { E } \cup \mathcal { V } ) } \stackrel { ( 2 . 3 ) } { \leq } F \left(\left\{z_{\alpha}^{n, w}\right\}_{\alpha \in \mathcal{E} \cup \mathcal{V})}-\frac{1}{2}\left\|v_{A}^{n, w}-v_{A}^{n, w-1}\right\|^{2} .\right.\right.
$$

(The last term in (2.19) arises from the quadratic term in (2.3).) By line 10 of Algorithm 2.1, $z_{i}^{n+1,0}=z_{i}^{n, \bar{w}}$ for all $i \in \mathcal{V}$ and $v_{H}^{n+1,0}=v_{H}^{n, \bar{w}}$ (even though the decompositions (2.2d) of $v_{H}^{n+1,0}$ and $v_{H}^{n, \bar{w}}$ may be different). Combining (2.19) over all $m \in\{1, \ldots, n\}$ and $w \in\{1, \ldots, \bar{w}\}$, we have

$$
F\left(\left\{z_{\alpha}^{1,0}\right\}_{\alpha \in \mathcal{E} \cup \mathcal{V}}\right)+\sum_{m=1}^{n} \sum_{w=1}^{\bar{w}}\left\|v_{A}^{m, w}-v_{A}^{m, w-1}\right\|^{2} \stackrel{(2.19)}{\leq} F\left(\left\{z_{\alpha}^{n, \bar{w}}\right\}_{\alpha \in \mathcal{E} \cup \mathcal{V})}\right.
$$




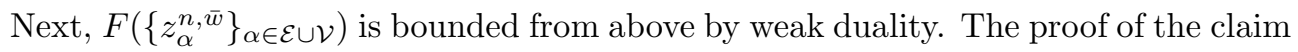
is complete.

Proof of (ii): Substituting $\left\{z_{\alpha}\right\}_{\alpha \in \mathcal{E} \cup \mathcal{V}}$ in (2.5) to be $\left\{z_{\alpha}^{n, w}\right\}_{\alpha \in \mathcal{E} \cup \mathcal{V}}$ and $x$ to be the primal minimizer $x^{*}$, we have

$$
\begin{array}{ll} 
& \frac{1}{2}\left\|x_{0}-x^{*}\right\|^{2}+\sum_{\alpha \in \mathcal{E} \cup \mathcal{V}} h_{\alpha}\left(x^{*}\right)-F\left(\left\{z_{\alpha}^{1,0}\right\}_{\alpha \in \mathcal{E} \cup \mathcal{V})}\right. \\
\stackrel{\text { part (i) }}{\geq} & \frac{1}{2}\left\|x_{0}-x^{*}\right\|^{2}+\sum_{\alpha \in \mathcal{E} \cup \mathcal{V}} h_{\alpha}\left(x^{*}\right)-F\left(\left\{z_{\alpha}^{n, w}\right\}_{\alpha \in \mathcal{E} \cup \mathcal{V})}\right. \\
\stackrel{(2.5)}{\geq} & \frac{1}{2}\left\|x_{0}-x^{*}-\sum_{\alpha \in \mathcal{E} \cup \mathcal{V}} z_{\alpha}^{n, w}\right\|^{2} \stackrel{(2.4 \mathrm{~b})}{=} \frac{1}{2}\left\|x_{0}-x^{*}-v_{A}^{n, w}\right\|^{2} .
\end{array}
$$

The conclusion is immediate.

Proof of (iii): We first make use of the technique in [5, Lemma 29.1] (which in turn is largely attributed to [12]) to show that

$$
\liminf _{n \rightarrow \infty}\left[\left(\sum_{w=1}^{\bar{w}}\left\|v_{A}^{n, w}-v_{A}^{n, w-1}\right\|\right) \sqrt{n}\right]=0
$$

Seeking a contradiction, suppose instead that there is an $\epsilon>0$ and $\bar{n}>0$ such that if $n>\bar{n}$, then $\left(\sum_{w=1}^{\bar{w}}\left\|v_{A}^{n, w}-v_{A}^{n, w-1}\right\|\right) \sqrt{n}>\epsilon$. By the Cauchy Schwarz inequality, we have $\frac{\epsilon^{2}}{n}<\left(\sum_{w=1}^{\bar{w}}\left\|v_{A}^{n, w}-v_{A}^{n, w-1}\right\|\right)^{2} \leq \bar{w} \sum_{w=1}^{\bar{w}}\left\|v_{A}^{n, w}-v_{A}^{n, w-1}\right\|^{2}$. This contradicts the earlier claim in (i) that $\sum_{n=1}^{\infty} \sum_{w=1}^{\bar{w}}\left\|v_{A}^{n, w}-v_{A}^{n, w-1}\right\|^{2}$ is finite.

Through (2.20), we find a sequence $\left\{n_{k}\right\}_{k=1}^{\infty}$ such that

$$
\lim _{k \rightarrow \infty}\left[\left(\sum_{w=1}^{\bar{w}}\left\|v_{A}^{n_{k}, w}-v_{A}^{n_{k}, w-1}\right\|\right) \sqrt{n_{k}}\right]=0 .
$$

Recalling the assumption (2.17), we get

$$
\lim _{k \rightarrow \infty}\left[\left(\sum_{w=1}^{\bar{w}}\left\|v_{A}^{n_{k}, w}-v_{A}^{n_{k}, w-1}\right\|\right)\left\|z_{\alpha}^{n_{k}, \bar{w}}\right\|\right] \stackrel{(2.17),(2.21)}{=} 0 \text { for all } \alpha \in \mathcal{E} \cup \mathcal{V}
$$

Moreover,

$$
\begin{aligned}
\left|\left\langle v_{A}^{n_{k}, \bar{w}}-v_{A}^{n_{k}, p\left(n_{k}, \alpha\right)}, z_{\alpha}^{n_{k}, \bar{w}}\right\rangle\right| & \leq\left\|v_{A}^{n_{k}, \bar{w}}-v_{A}^{n_{k}, p\left(n_{k}, \alpha\right)}\right\|\left\|z_{\alpha}^{n_{k}, \bar{w}}\right\| \\
& \leq\left(\sum_{w=1}^{\bar{w}}\left\|v_{A}^{n_{k}, w}-v_{A}^{n_{k}, w-1}\right\|\right)\left\|z_{\alpha}^{n_{k}, \bar{w}}\right\| .
\end{aligned}
$$

By (ii) and the finite dimensionality of $X$, there exists a further subsequence of $\left\{v_{A}^{n_{k}, \bar{w}}\right\}_{k=1}^{\infty}$ which converges to some $v_{A}^{*} \in X$. Combining (2.22) and (2.23) gives (iii). 
Proof of (iv): From earlier results, we obtain

$$
-\sum_{\alpha \in \mathcal{E} \cup \mathcal{V}} h_{\alpha}\left(x_{0}-v_{A}^{*}\right)
$$

$$
\begin{aligned}
& (2.5) \\
& \frac{1}{2}\left\|x_{0}-\left(x_{0}-v_{A}^{*}\right)\right\|^{2}-F\left(\left\{z_{\alpha}^{n_{k}, \bar{w}}\right\}_{\alpha \in \mathcal{E} \cup \mathcal{V})}\right. \\
& \text { (1.8), } \stackrel{(2.15)}{=} \\
& \frac{1}{2}\left\|v_{A}^{*}\right\|^{2}+\sum_{\alpha \in \mathcal{E}_{n_{k}} \cup \mathcal{V}} h_{\alpha}^{*}\left(z_{\alpha}^{n_{k}, p\left(n_{k}, \alpha\right)}\right) \\
& +\sum_{(i, j) \notin \mathcal{E}_{n_{k}}} h_{(i, j)}^{*}\left(z_{(i, j)}^{n_{k}, \bar{w}}\right)-\left\langle x_{0}, v_{A}^{n_{k}, \bar{w}}\right\rangle+\frac{1}{2}\left\|v_{A}^{n_{k}, \bar{w}}\right\|^{2} \\
& \text { Claim 2.6(c), } \stackrel{\in \in S_{n, p(n, \alpha)},(2.16)}{=} \frac{1}{2}\left\|v_{A}^{*}\right\|^{2}+\sum_{\alpha \in \mathcal{E}_{n_{k}} \cup \mathcal{V}}\left\langle x_{0}-v_{A}^{n_{k}, p\left(n_{k}, \alpha\right)}, z_{\alpha}^{n_{k}, p\left(n_{k}, \alpha\right)}\right\rangle \\
& -\sum_{\alpha \in \mathcal{E}_{n_{k}} \cup \mathcal{V}} h_{\alpha}\left(x_{0}-v_{A}^{n_{k}, p\left(n_{k}, \alpha\right)}\right)-\left\langle x_{0}, v_{A}^{n_{k}, \bar{w}}\right\rangle+\frac{1}{2}\left\|v_{A}^{n_{k}, \bar{w}}\right\|^{2} \\
& \stackrel{(2.15)}{=} \quad \frac{1}{2}\left\|v_{A}^{*}\right\|^{2}-\sum_{\alpha \in \mathcal{E}_{n_{k}} \cup \mathcal{V}}\left\langle v_{A}^{n_{k}, p\left(n_{k}, \alpha\right)}-v_{A}^{n_{k}, \bar{w}}, z_{\alpha}^{n_{k}, \bar{w}}\right\rangle \\
& -\sum_{\alpha \in \mathcal{E}_{n_{k}} \cup \mathcal{V}} h_{\alpha}\left(x_{0}-v_{A}^{n_{k}, p\left(n_{k}, \alpha\right)}\right)-\left\langle x_{0}, v_{A}^{n_{k}, \bar{w}}\right\rangle \\
& +\left\langle x_{0}-v_{A}^{n_{k}, \bar{w}}, \sum_{\alpha \in \mathcal{E}_{n_{k}} \cup \mathcal{V}} z_{\alpha}^{n_{k}, p\left(n_{k}, \alpha\right)}\right\rangle+\frac{1}{2}\left\|v_{A}^{n_{k}, \bar{w}}\right\|^{2} \\
& (2.4 \mathrm{~b}),(2.16) \\
& \frac{1}{2}\left\|v_{A}^{*}\right\|^{2}-\frac{1}{2}\left\|v_{A}^{n_{k}, \bar{w}}\right\|^{2}-\sum_{\alpha \in \mathcal{E}_{n_{k}} \cup \mathcal{V}}\left\langle v_{A}^{n_{k}, p\left(n_{k}, \alpha\right)}-v_{A}^{n_{k}, \bar{w}}, z_{\alpha}^{n_{k}, \bar{w}}\right\rangle \\
& -\sum_{\alpha \in \mathcal{E}_{n_{k}} \cup \mathcal{V}} h_{\alpha}\left(x_{0}-v_{A}^{n_{k}, p\left(n_{k}, \alpha\right)}\right)
\end{aligned}
$$

Since $\lim _{k \rightarrow \infty} v_{A}^{n_{k}, \bar{w}}=v_{A}^{*}$, we have $\lim _{k \rightarrow \infty} \frac{1}{2}\left\|v_{A}^{*}\right\|^{2}-\frac{1}{2}\left\|v_{A}^{n_{k}, \bar{w}}\right\|^{2}=0$. The third term in the last group of formulas (i.e., the sum involving the inner products) converges to 0 by (iii).

Next, recall that if $(i, j) \in \mathcal{E}_{n}$, by $(2.6)$, we have $h_{(i, j)}\left(x_{0}-v_{A}^{n, p(n,(i, j))}\right)=0$, which gives $x_{0}-v_{A}^{n, p(n,(i, j))} \stackrel{(1.2),(1.1)}{\in} H_{(i, j)}$. There is a constant $\kappa_{\mathcal{E}_{n_{k}}}>0$ such that

$$
\begin{array}{cl} 
& d\left(x_{0}-v_{A}^{n_{k}, \bar{w}}, \cap_{(i, j) \in \mathcal{E}} H_{(i, j)}\right) \\
\mathcal{E}_{n_{k}} \text { connects } \mathcal{V}, \text { Prop 2.1(1) } & d\left(x_{0}-v_{A}^{n_{k}, \bar{w}}, \cap_{(i, j) \in \mathcal{E}_{n_{k}}} H_{(i, j)}\right) \\
\leq & \kappa_{\mathcal{E}_{n_{k}}} \max _{(i, j) \in \mathcal{E}_{n_{k}}} d\left(x_{0}-v_{A}^{n_{k}, \bar{w}}, H_{(i, j)}\right) \\
x_{0}-v_{A}^{n_{k}, p\left(n_{k},(i, j)\right)} \in H_{(i, j)} & \\
\leq & \kappa_{\mathcal{E}_{n_{k}}} \max _{(i, j) \in \mathcal{E}_{n_{k}}}\left\|v_{A}^{n_{k}, \bar{w}}-v_{A}^{n_{k}, p\left(n_{k},(i, j)\right)}\right\| .
\end{array}
$$

Let $\kappa:=\max \left\{\kappa_{\mathcal{E}^{\prime}}: \mathcal{E}^{\prime}\right.$ connects $\left.\mathcal{V}\right\}$. We have $\kappa_{\mathcal{E}_{n_{k}}} \leq \kappa$. Taking limits of (2.25), the RHS converges to zero by (i), so $d\left(x_{0}-v_{A}^{*}, \cap_{(i, j) \in \mathcal{E}} H_{(i, j)}\right)=0$, or $x_{0}-v_{A}^{*} \in$ $\cap_{(i, j) \in \mathcal{E}} H_{(i, j)}$. So $\sum_{(i, j) \in \mathcal{E}} h_{(i, j)}\left(x_{0}-v_{A}^{*}\right)=0$. Together with the fact that $x_{0}-$ $v_{A}^{n_{k}, p\left(n_{k},(i, j)\right)} \in H_{(i, j)}$, we have

$$
\sum_{(i, j) \in \mathcal{E}_{n_{k}}} h_{(i, j)}\left(x_{0}-v_{A}^{n_{k}, p\left(n_{k},(i, j)\right)}\right)=0=\sum_{(i, j) \in \mathcal{E}} h_{(i, j)}\left(x_{0}-v_{A}^{*}\right) .
$$


Lastly, by the lower semicontinuity of $h_{i}(\cdot)$, we have

$$
-\lim _{k \rightarrow \infty} \sum_{i \in \mathcal{V}} h_{i}\left(x_{0}-v_{A}^{n_{k}, p\left(n_{k}, i\right)}\right) \leq-\sum_{i \in \mathcal{V}} h_{i}\left(x_{0}-v_{A}^{*}\right) .
$$

As mentioned after (2.24), taking the limits as $k \rightarrow \infty$ would result in the first three terms of the last formula in (2.24) to be zero. Hence

$$
\begin{gathered}
-\sum_{\alpha \in \mathcal{E} \cup \mathcal{V}} h_{\alpha}\left(x_{0}-v_{A}^{*}\right) \stackrel{(2.24)}{\leq} \lim _{k \rightarrow \infty}-\sum_{\alpha \in \mathcal{E}_{n_{k}} \cup \mathcal{V}} h_{\alpha}\left(x_{0}-v_{A}^{n_{k}, p\left(n_{k}, \alpha\right)}\right) \\
\stackrel{(2.26),(2.27)}{\leq}-\sum_{\alpha \in \mathcal{E} \cup \mathcal{V}} h_{\alpha}\left(x_{0}-v_{A}^{*}\right) .
\end{gathered}
$$

So (2.24) becomes an equation in the limit. The first two lines of (2.24) then gives

$$
\lim _{k \rightarrow \infty} F\left(\left\{z_{\alpha}^{n_{k}, \bar{w}}\right\}_{\alpha \in \mathcal{E} \cup \mathcal{V})}=\frac{1}{2}\left\|v_{A}^{*}\right\|^{2}+\sum_{i \in \mathcal{V}} h_{i}\left(x_{0}-v_{A}^{*}\right),\right.
$$

which shows that $x_{0}-v_{A}^{*}$ is the primal minimizer.

A last detail that we need to resolve is to show that (2.17) holds for the choice of $S_{n, w}$ in Algorithm 2.1.

Proposition 2.15. (Growth of $\sum_{\alpha \in \mathcal{E} \cup \mathcal{V}}\left\|z_{\alpha}^{n, w}\right\|$ ) If $S_{n, w}$ are such that $\mid S_{n, w} \cap$ $\mathcal{V} \mid \leq 1$ for all $n \in \mathbb{N}$ and $w \in\{1, \ldots, \bar{w}\}$ like in Algorithm 2.1, then (2.17) holds.

Proof. We either have $S_{n, w} \cap \mathcal{V}=\emptyset$ or $\left|S_{n, w} \cap \mathcal{V}\right|=1$. In the second case, let $i^{*}$ be the index such that $i^{*} \in S_{n, w} \cap \mathcal{V}$. Otherwise, in the first case, we let $i^{*}$ be any index in $\mathcal{V}$. We have

$$
\begin{aligned}
\sum_{i \in \mathcal{V}}\left[v_{A}^{n, w}-v_{A}^{n, w-1}\right]_{i} & \stackrel{\text { line }}{\stackrel{8,(2.4)}{=}} \sum_{i \in \mathcal{V}} \sum_{\alpha \in S_{n, w}}\left[z_{\alpha}^{n, w}-z_{\alpha}^{n, w-1}\right]_{i} \\
z_{(i, j)} & \stackrel{D^{\perp},(2.1)}{=} \sum_{i \in \mathcal{V}}\left[z_{i^{*}}^{n, w}-z_{i^{*}}^{n, w-1}\right]_{i} \\
\stackrel{\text { Prop }}{=} 2.7 & {\left[z_{i^{*}}^{n, w}-z_{i^{*}}^{n, w-1}\right]_{i *} . }
\end{aligned}
$$

Recall that the norm $\|\cdot\|$ always refers to the 2-norm unless stated otherwise. By the equivalence of norms in finite dimensions, we can find a constant $c_{1}$ such that

$$
\begin{aligned}
\left\|v_{A}^{n, w}-v_{A}^{n, w-1}\right\| & \geq c_{1} \sum_{i \in \mathcal{V}}\left\|\left[v_{A}^{n, w}-v_{A}^{n, w-1}\right]_{i}\right\| \\
& \geq c_{1}\left\|\sum_{i \in \mathcal{V}}\left[v_{A}^{n, w}-v_{A}^{n, w-1}\right]_{i}\right\| \\
\stackrel{(2.28)}{=} & c_{1}\left\|z_{i^{*}}^{n, w}-z_{i^{*}}^{n, w-1}\right\| \stackrel{(2.3)}{=} c_{1} \sum_{i \in \mathcal{V}}\left\|z_{i}^{n, w}-z_{i}^{n, w-1}\right\| .
\end{aligned}
$$

Next, $v_{H}^{n, w}-v_{H}^{n, w-1} \stackrel{(2.4 \mathrm{~b})}{=} v_{A}^{n, w}-v_{A}^{n, w-1}-\left(z_{i^{*}}^{n, w}-z_{i^{*}}^{n, w-1}\right)$, so

$$
\begin{aligned}
\left\|v_{H}^{n, w}-v_{H}^{n, w-1}\right\| & \leq\left\|v_{A}^{n, w}-v_{A}^{n, w-1}\right\|+\left\|z_{i^{*}}^{n, w}-z_{i^{*}}^{n, w-1}\right\| \\
& \stackrel{(2.29)}{\leq}\left(1+\frac{1}{c_{1}}\right)\left\|v_{A}^{n, w}-v_{A}^{n, w-1}\right\| .
\end{aligned}
$$


We can choose $\left\{z_{(i, j)}^{n, w}\right\}_{(i, j) \in \mathcal{E}}$ such that

$$
\sum_{(i, j) \in S_{n, w} \cap \mathcal{E}}\left[z_{(i, j)}^{n, w}-z_{(i, j)}^{n, w-1}\right] \stackrel{\text { line }}{=} \sum_{(i, j) \in \mathcal{E}}\left[z_{(i, j)}^{n, w}-z_{(i, j)}^{n, w-1}\right] \stackrel{(2,4 a)}{=} v_{H}^{n, w}-v_{H}^{n, w-1} .
$$

Without loss of generality, we can assume that $S_{n, w} \cap \mathcal{E}$ contains edges that do not form a cycle. This also means that for a $v_{H}^{n, w}-v_{H}^{n, w-1}$, each $z_{(i, j)}^{n, w}-z_{(i, j)}^{n, w-1}$ can be determined uniquely with a linear map from the relation (2.31). Therefore there is a constant $\kappa_{(i, j), S_{n, w} \cap \mathcal{E}}>0$ such that

$$
\left\|z_{(i, j)}^{n, w}-z_{(i, j)}^{n, w-1}\right\| \leq \kappa_{(i, j), S_{n, w} \cap \mathcal{E}}\left\|v_{H}^{n, w}-v_{H}^{n, w-1}\right\| .
$$

Thus there is a constant $\kappa>0$ such that

$$
\sum_{(i, j) \in \mathcal{E}}\left\|z_{(i, j)}^{n, w}-z_{(i, j)}^{n, w-1}\right\| \stackrel{(2.3)}{=} \sum_{(i, j) \in S_{n, w} \cap \mathcal{E}}\left\|z_{(i, j)}^{n, w}-z_{(i, j)}^{n, w-1}\right\| \stackrel{(2.32)}{\leq} \kappa\left\|v_{H}^{n, w}-v_{H}^{n, w-1}\right\| .
$$

Combining (2.29), (2.30) and (2.33) together shows that there is a constant $C_{2}>1$ such that

(2.34)

$\left\|v_{H}^{n, w}-v_{H}^{n, w-1}\right\|+\sum_{(i, j) \in \mathcal{E}}\left\|z_{(i, j)}^{n, w}-z_{(i, j)}^{n, w-1}\right\|+\sum_{i \in \mathcal{V}}\left\|z_{i}^{n, w}-z_{i}^{n, w-1}\right\| \leq C_{2}\left\|v_{A}^{n, w}-v_{A}^{n, w-1}\right\|$.

Since $\left\{z_{\alpha}^{n, 0}\right\}_{\alpha \in \mathcal{E}}$ was chosen to satisfy (2.2), there is some $M>1$ such that

$$
\sum_{\alpha \in \mathcal{E}}\left\|z_{\alpha}^{n, 0}\right\| \stackrel{(2.2 \mathrm{c})}{\leq} M\left\|v_{H}^{n, 0}\right\| \stackrel{(2.2 \mathrm{~d})}{\leq} M\left(\left\|v_{H}^{1,0}\right\|+\sum_{m=1}^{n-1} \sum_{w=1}^{\bar{w}}\left\|v_{H}^{m, w}-v_{H}^{m, w-1}\right\|\right)
$$

Now for any $n \geq 1$, we have

$$
\begin{aligned}
(2.36) \sum_{\alpha \in \mathcal{E} \cup \mathcal{V}}\left\|z_{\alpha}^{n, \bar{w}}\right\| \leq & \sum_{\alpha \in \mathcal{E}}\left\|z_{\alpha}^{n, 0}\right\|+\sum_{w=1}^{\bar{w}} \sum_{\alpha \in \mathcal{E}}\left\|z_{\alpha}^{n, w}-z_{\alpha}^{n, w-1}\right\| \\
& +\sum_{m=1}^{n} \sum_{w=1}^{\bar{w}} \sum_{\alpha \in \mathcal{V}}\left\|z_{\alpha}^{m, w}-z_{\alpha}^{m, w-1}\right\|+\sum_{\alpha \in \mathcal{V}}\left\|z_{\alpha}^{1,0}\right\| \\
\stackrel{(2.35)}{\leq} & M\left\|v_{H}^{1,0}\right\|+\sum_{\alpha \in \mathcal{V}}\left\|z_{\alpha}^{1,0}\right\|+\sum_{w=1}^{\bar{w}}\left(\sum_{\alpha \in \mathcal{E}}\left\|z_{\alpha}^{n, w}-z_{\alpha}^{n, w-1}\right\|\right) \\
& +\sum_{m=1}^{n-1} \sum_{w=1}^{\bar{w}}\left(M\left\|v_{H}^{m, w}-v_{H}^{m, w-1}\right\|+\sum_{\alpha \in \mathcal{V}}\left\|z_{\alpha}^{m, w}-z_{\alpha}^{m, w-1}\right\|\right) \\
\stackrel{(2.34)}{\leq} & M\left\|v_{H}^{1,0}\right\|+\sum_{\alpha \in \mathcal{V}}\left\|z_{\alpha}^{1,0}\right\|+M C_{2} \sum_{m=1}^{n} \sum_{w=1}^{\bar{w}}\left\|v_{A}^{n, w}-v_{A}^{n, w-1}\right\| .
\end{aligned}
$$

By the Cauchy Schwarz inequality, we have

$$
\sum_{m=1}^{n} \sum_{w=1}^{\bar{w}}\left\|v_{A}^{m, w}-v_{A}^{m, w-1}\right\| \leq \sqrt{n \bar{w}} \sqrt{\sum_{m=1}^{n} \sum_{w=1}^{\bar{w}}\left\|v_{A}^{m, w}-v_{A}^{m, w-1}\right\|^{2} .}
$$

Since the second square root of the right hand side of (2.37) is bounded by Theorem 2.14(i), we make use of (2.36) to obtain the conclusion (2.17) as needed. 
Remark 2.16. (Convergence rate) An aspect of Algorithm 2.1 that we do not cover in this paper is the convergence rate. In the case where there are no dual minimizers, components of the dual variables $\left\{\left\{z_{\alpha}^{n, w}\right\}_{\alpha \in \mathcal{E} \cup \mathcal{V}}\right\}_{\substack{1 \leq n<\infty \\ 0 \leq w \leq w}}$ need not be bounded. But in the case where the variables $\left\{\left\{z_{\alpha}^{n, w}\right\}_{\alpha \in \mathcal{E} \cup \mathcal{V}}\right\}_{\substack{1 \leq n \leq \infty \\ 0 \leq w \leq w}}$ remain bounded as $n \rightarrow \infty$, an $O(1 / n)$ rate was shown for the dual objective function, which leads to an $O(1 / \sqrt{n})$ convergence rate of the distance $\left\|x^{n, w}-x^{*}\right\|$ to the optimal solution $x^{*}$. The ideas for these results are presented in [38, Section 3]. Such ideas were already present in $[8,6]$ for example.

2.5. Generality of (1.5). Another case of interest is when $\frac{1}{2}\left\|x-x_{0}\right\|^{2}$ in (1.5) is replaced by $\frac{1}{2}\left\|x-x_{0}\right\|_{Q}^{2}$, where where $\|x\|_{Q}^{2}=\langle x, Q x\rangle$ and $Q$ is a block diagonal positive definite matrix. In the case where $Q$ is such that $\|x\|_{Q}^{2}=\sum_{i \in \mathcal{V}} \lambda_{i}\left\|x_{i}\right\|^{2}$ for some $\lambda \in \mathbb{R}^{|\mathcal{V}|}$ such that $\lambda>0$ and $\mathbf{f}_{i} \equiv 0$ for all $i \in \mathcal{V}$, then the minimizer of (1.5) is $\frac{1}{\sum_{i \in \mathcal{V}} \lambda_{i}} \sum_{i \in \mathcal{V}} \lambda_{i} y_{i}^{0}$. In other words, (1.5) becomes a weighted average consensus problem.

We show how to transform a problem involving $\frac{1}{2}\left\|x-x_{0}\right\|_{Q}^{2}$ to one involving $\frac{1}{2}\left\|x-x_{0}\right\|^{2}$. Note that

$$
\begin{aligned}
& \min _{x \in X^{|\mathcal{V}|}} \frac{1}{2}\left\|x-x_{0}\right\|_{Q}^{2}+\sum_{(i, j) \in \mathcal{E}} \delta_{H_{(i, j)}}(x)+\sum_{i \in \mathcal{V}} \mathbf{f}_{i}(x) \\
\equiv & \min _{x \in X^{|\mathcal{V}|}} \frac{1}{2}\left\|Q^{1 / 2} x-Q^{1 / 2} x_{0}\right\|^{2}+\sum_{(i, j) \in \mathcal{E}} \delta_{Q^{1 / 2} H_{(i, j)}}\left(Q^{1 / 2} x\right)+\sum_{i \in \mathcal{V}} \mathbf{f}_{i} \circ Q^{-1 / 2}\left(Q^{1 / 2} x\right) .
\end{aligned}
$$

We can thus let $v^{0}$ be $Q^{1 / 2} x_{0}$, and seek the variable $v=Q^{1 / 2} x$. The function $\delta_{Q^{1 / 2} H_{(i, j)}}(\cdot)$ requires a transformation of the set (1.1), but the transformed problem would fit the framework of Dykstra's algorithm.

3. Distributed algorithm for functions not strongly convex. We saw earlier that the minimization of the sum of strongly convex functions can be minimized over a network. A natural question to ask is whether it is possible to minimize the sum of functions that are not necessarily strongly convex in the same setting.

A technique for minimizing (1.4) is to choose 2 or more nodes, say $S_{k} \subset \mathcal{V}$ (which preferably forms a connected subgraph to allow for communications), and then minimize the function varying only the dual variables corresponding to the chosen nodes. This leads to Algorithm 3.1.

Such a method is analogous to the method of alternating minimization, which have stationary points that are not optimal points. We now show an example of such a stationary point for Algorithm 3.1.

Example 3.1. (Algorithm 3.1 can get stuck at non-optimal value) Consider a graph with $\mathcal{V}=\{1,2,3\}$ and $\mathcal{E}=\{(1,2),(2,3)\}$. Let $f_{i}: \mathbb{R} \rightarrow \overline{\mathbb{R}}, i \in\{1,2,3\}$, be defined by

$$
f_{1}(x)=\frac{1}{2}(x+1)^{2}, \quad f_{2}(x)=\delta_{\{0\}}(x), \quad f_{3}(x)=\frac{1}{2}(x-1)^{2},
$$

and we have $f_{1}^{*}(z)=\frac{1}{2} z^{2}-z, f_{2}^{*}(z)=\delta_{\mathbb{R}}(z)$ and $f_{3}^{*}(z)=\frac{1}{2} z^{2}+z$. Let $\bar{y}=(0,0,0) \in$ 
Algorithm 3.1 Distributed dual ascent algorithm

Consider the problem (1.4).

Let $\left\{y_{i}^{0}\right\}_{i \in \mathcal{V}} \subset X$ be starting variables such that

$$
\sum_{i \in \mathcal{V}} y_{i}^{0}=0
$$

For $n=1, \ldots$

Find a set $S_{n} \subset \mathcal{V}$ such that $\left|S_{n}\right| \geq 2$.

For all $i \in S_{n}$, define $y_{i}^{n}$ so that

$$
\begin{aligned}
\left\{y_{i}^{n}\right\}_{i \in S_{n}} \in \underset{\left\{y_{i}\right\}_{i \in S_{n}}}{\arg \max }-\sum_{i \in S_{n}} f_{i}^{*}\left(y_{i}\right) \\
\text { s.t. } \quad \sum_{i \in S_{n}} y_{i}=\sum_{i \in S_{n}} y_{i}^{n-1} .
\end{aligned}
$$

Define $y_{i}^{k}=y_{i}^{k-1}$ for all $i \notin S_{n}$.

End for

$\mathbb{R}^{3}$. For $S \subset\{1,2,3\}$, denote the dual problem $\left(D P_{S}\right)$ by

$$
\begin{aligned}
\left(D P_{S}\right) & \max _{y_{i} \in \mathbb{R}, i \in S}-\sum_{i \in S} f_{i}^{*}\left(y_{i}\right) \\
\text { s.t. } \quad & \sum_{i \in S} y_{i}=\sum_{i \in S} \bar{y}_{i} .
\end{aligned}
$$

The problem $\left(D P_{\{1,2\}}\right)$ has minimizer $y_{1}=y_{2}=0$ with $-1 \in \partial f_{1}^{*}(0)$ and $-1 \in \partial f_{2}^{*}(0)$, and the problem $\left(D P_{\{2,3\}}\right)$ minimizer $y_{2}=y_{3}=0$ with $1 \in \partial f_{2}^{*}(0)$ and $1 \in \partial f_{3}^{*}(0)$. Hence the problem $\left(D P_{\{1,2,3\}}\right)$ has a stationary point of $\bar{y}=(0,0,0)$ for the alternating minimization method. However, the global minimizer to $\left(D P_{\{1,2,3\}}\right)$ is $(1,0,-1)$ with $0 \in \partial f_{i}^{*}\left(y_{i}\right)$ for $i \in\{1,2,3\}$.

Note from this example that the failure can be identified from the fact that $x=-1$ if we use the edge $(1,2)$ and $x=1$ if we use the edge $(2,3)$. Node 2 should be able to figure out that the $x$ values corresponding to edges $(1,2)$ and $(2,3)$ are too far apart, and one needs to minimize $f_{1}^{*}(\cdot)+f_{3}^{*}(\cdot)$ in order to avoid convergence to a non optimal value.

We now give a proof for the convergence of Algorithm 3.1, which is based on the proof in [44] (and who in turn cited other references). To shorten notation, for each set $S \subset \mathcal{V}$, we let $\mathcal{D}(S)$ be the set of directions $d$ in $X^{|\mathcal{V}|}$ defined by

$$
\mathcal{D}(S):=\left\{d \in X^{|\mathcal{V}|}: \sum_{i=1}^{|\mathcal{V}|} d_{i}=0 \text { and } \operatorname{supp}(d) \subset S\right\},
$$

where $\operatorname{supp}(d)$ is the set $\left\{i: d_{i} \neq 0\right\}$. We define $G: X^{|\mathcal{V}|} \rightarrow \overline{\mathbb{R}}$ by

$$
G(y)=\sum_{i \in \mathcal{V}} f_{i}^{*}\left(y_{i}\right)
$$

and let $G^{\prime}(y ; d)$ be the directional derivative of $G$ at $y$ in the direction $d$. 
TheOREm 3.2. (Convergence of Algorithm 3.1) Suppose that there is an integer $T$ such that for every $n \geq 1$, the sets $\left\{S_{n+i}\right\}_{i=1}^{T}$ satisfies the following:

(a) Suppose $S^{\prime}, S^{\prime \prime}$ are elements in $\left\{S_{n+i}\right\}_{i=1}^{T}$ such that $S^{\prime} \cap S^{\prime \prime} \neq \emptyset$ and $y \in X^{|\mathcal{V}|}$. Then for all $d \in \mathcal{D}\left(S^{\prime} \cup S^{\prime \prime}\right)$, we can find $d^{\prime} \in \mathcal{D}\left(S^{\prime}\right)$ and $d^{\prime \prime} \in \mathcal{D}\left(S^{\prime \prime}\right)$ such that $d=d^{\prime}+d^{\prime \prime}$ and

$$
G^{\prime}(y ; d)=G^{\prime}\left(y ; d^{\prime}\right)+G^{\prime}\left(y ; d^{\prime \prime}\right) .
$$

(b) Suppose $y \in X^{|\mathcal{V}|}$. If for all $r \in\{1, \ldots, T\}, G^{\prime}\left(y ; d_{r}\right) \geq 0$ for all $d_{r} \in$ $\mathcal{D}\left(S_{n+r}\right)$, then $y$ is a minimizer of $G(\cdot)$.

Suppose further that the sequence $\left\{y^{n}\right\}_{n=1}^{\infty}$ is bounded. Then every cluster point of $\left\{y^{n}\right\}_{n=1}^{\infty}$ is a minimizer of $G$.

Proof. Our proof is adapted from the ideas in [44, Section 4]. Suppose $\bar{y} \in X^{|\mathcal{V}|}$ is a cluster point of $\left\{y^{n}\right\}_{n=1}^{\infty}$ and that $\mathcal{R} \subset\{1,2, \ldots\}$ is such that $\lim _{r \in \mathcal{R}} y^{r}=\bar{y}$. We can assume, by taking subsequences if necessary, that $\lim _{r \in \mathcal{R}} y^{r-T+1+j}$ converges to some $\bar{y}^{j}$ for all $j \in\{1, \ldots, T\}$. We have $\bar{y}^{T-1}=\bar{y}$. We also note that $\left\{G\left(y^{n}\right)\right\}_{n=1}^{\infty}$ is a non-increasing sequence, so

$$
\lim _{r \in \mathcal{R}} G\left(y^{r-T+1+j}\right) \text { exists for all } j \in\{1, \ldots, T\} .
$$

Next, we can assume that for each $j \in\{1, \ldots, T\}$, the set $\left\{S_{r-T+1+j}\right\}_{r \in \mathcal{R}}$ depends only on $j$, which we call $\bar{S}_{j}$. For each $j \in\{1, \ldots, T\}$, since $\bar{S}_{j}$ is chosen at iteration $r-T+1+j$ for $r \in \mathcal{R}$, we have

$$
\begin{aligned}
G\left(y^{r-T+1+j}\right) & \leq G\left(y^{r-T+1+j}+d_{j}\right) \text { for all } d_{j} \in \mathcal{D}\left(\bar{S}_{j}\right) \\
y_{i}^{r-T+j} & =y_{i}^{r-T+1+j} \text { for all } i \notin \bar{S}_{j} .
\end{aligned}
$$

Then the continuity of $G(\cdot)$ gives us

$$
\begin{aligned}
G\left(\bar{y}^{j}\right) & \leq G\left(\bar{y}^{j}+d_{j}\right) \text { for all } d_{j} \in \mathcal{D}\left(\bar{S}_{j}\right) \\
\bar{y}_{i}^{j-1} & =\bar{y}_{i}^{j} \text { for all } i \notin \bar{S}_{j} .
\end{aligned}
$$

We have $G\left(\bar{y}^{1}\right)=\cdots=G\left(\bar{y}^{T}\right)$. The previous line also gives $\bar{y}^{j}-\bar{y}^{j-1} \in \mathcal{D}\left(\bar{S}_{j}\right)$, so

$$
G\left(\bar{y}^{j-1}\right)=G\left(\bar{y}^{j}\right) \stackrel{(3.3)}{\leq} G\left(\bar{y}^{j}+\left(d_{j}+\bar{y}^{j-1}-\bar{y}^{j}\right)\right)=G\left(\bar{y}^{j-1}+d_{j}\right) \text { for all } d_{j} \in \mathcal{D}\left(\bar{S}_{j}\right) .
$$

We claim that for $j=1, \ldots, T-1$,

$$
G\left(\bar{y}^{j}\right) \leq G\left(\bar{y}^{j}+d_{k}\right) \text { for all } d_{k} \in \mathcal{D}\left(\bar{S}_{1}\right), \mathcal{D}\left(\bar{S}_{2}\right), \ldots \text {, or } \mathcal{D}\left(\bar{S}_{j}\right) .
$$

By (3.3), (3.5) holds for $j=1$. Suppose (3.5) holds for $j=1, \ldots, l-1$ for some $l \in\{2, \ldots, T-1\}$. We show that (3.5) holds for $j=l$. From (3.4),

$$
G\left(\bar{y}^{l-1}\right) \leq G\left(\bar{y}^{l-1}+d_{l}\right) \text { for all } d_{l} \in \mathcal{D}\left(\bar{S}_{l}\right),
$$

implying that

$$
G^{\prime}\left(\bar{y}^{l-1} ; \bar{y}^{l}-\bar{y}^{l-1}+v\right) \geq 0 \text { for all } v \in \mathcal{D}\left(\bar{S}_{l}\right) .
$$

Also, since (3.5) holds for $j=l-1$, we have, for all $k \in\{1, \ldots, l-1\}$,

$$
G\left(\bar{y}^{l-1}\right) \leq G\left(\bar{y}^{l-1}+d_{k}-v\right) \text { for all } d_{k} \in \mathcal{D}\left(\bar{S}_{k}\right) \text { and } v \in \mathcal{D}\left(\bar{S}_{l} \cap \bar{S}_{k}\right),
$$


which in turn implies

$$
G^{\prime}\left(\bar{y}^{l-1} ; d_{k}-v\right) \geq 0 \text { for all } d_{k} \in \mathcal{D}\left(\bar{S}_{k}\right) \text { and } v \in \mathcal{D}\left(\bar{S}_{l} \cap \bar{S}_{k}\right) .
$$

If $\bar{S}_{l} \cap \bar{S}_{k}=\emptyset$, then $v$ can be taken to be zero, and we get (3.1). By (3.6) and (3.7) and property (a) for each $d_{k}$, we can choose $v$ such that for all $d_{k} \in \mathcal{D}\left(\bar{S}_{k}\right)$

$$
G^{\prime}\left(\bar{y}^{l-1} ; \bar{y}^{l}-\bar{y}^{l-1}+d_{k}\right) \stackrel{\text { ppty }}{=}{ }^{\text {a })} G^{\prime}\left(\bar{y}^{l-1} ; \bar{y}^{l}-\bar{y}^{l-1}+v\right)+G^{\prime}\left(\bar{y}^{l-1} ; d_{k}-v\right) \stackrel{(3.6),(3.7)}{\geq} 0 .
$$

Since $G(\cdot)$ is convex,

$$
G\left(\bar{y}^{l}+d_{k}\right)=G\left(\bar{y}^{l-1}+\left(\bar{y}^{l}-\bar{y}^{l-1}+d_{k}\right)\right) \stackrel{(3.8)}{\geq} G\left(\bar{y}^{l-1}\right)=G\left(\bar{y}^{l}\right) \text { for all } d_{k} \in \mathcal{D}\left(\bar{S}_{k}\right) .
$$

Since (3.3) holds with $j=l,(3.5)$ holds for $j=l$. So (3.5) holds for all $j \in\{1, \ldots, T-$ 1 \}. Taking $j=T-1$ for (3.5) and combining property (b) proves that $\bar{y}=\bar{y}^{T-1}$ is a minimizer of $G(\cdot)$.

Define $\mathcal{V}_{s m} \subset \mathcal{V}$ to be such that

$$
\mathcal{V}_{s m}:=\left\{i \in \mathcal{V}: f_{i}^{*}(\cdot) \text { is smooth }\right\} .
$$

We give more insight on Properties (a) and (b) in Theorem 3.2.

Proposition 3.3. Property (a) in Theorem 3.2 is satisfied if for any two elements $S^{\prime}, S^{\prime \prime}$ in $\left\{S_{n+i}\right\}_{i=1}^{T}$, either $S^{\prime} \cap S^{\prime \prime}=\emptyset$ or $S^{\prime} \cap S^{\prime \prime} \cap \mathcal{V}_{s m} \neq \emptyset$.

Proof. We only need to consider the case when $S^{\prime} \cap S^{\prime \prime} \cap \mathcal{V}_{s m} \neq \emptyset$. We want to show that if $d \in \mathcal{D}\left(S^{\prime} \cup S^{\prime \prime}\right)$, then $d$ can be written as $d=d^{\prime}+d^{\prime \prime}$, where $d^{\prime} \in \mathcal{D}\left(S^{\prime}\right)$ and $d^{\prime \prime} \in \mathcal{D}\left(S^{\prime \prime}\right)$, so that (3.1) holds. If $S^{\prime} \cap S^{\prime \prime} \cap \mathcal{V}_{s m} \neq \emptyset$, then let $\bar{i} \in S^{\prime} \cap S^{\prime \prime} \cap \mathcal{V}_{s m}$. For a given $d \in \mathcal{D}\left(S^{\prime} \cup S^{\prime \prime}\right)$, define $d^{\prime}$ and $d^{\prime \prime}$ so that

$$
d_{i}^{\prime}=\left\{\begin{array}{ll}
d_{i} & \text { if } i \in S^{\prime} \backslash\{\bar{i}\} \\
-\sum_{i \in S^{\prime} \backslash\{\bar{i}\}} d_{i} & \text { if } i=\bar{i} \\
0 & \text { otherwise }
\end{array} \text { and } d_{i}^{\prime \prime}= \begin{cases}d_{i} & \text { if } i \in S^{\prime \prime} \backslash S^{\prime} \\
-\sum_{i \in S^{\prime \prime} \backslash S^{\prime}} d_{i} & \text { if } i=\bar{i} \\
0 & \text { otherwise. }\end{cases}\right.
$$

It is clear to see that $d=d^{\prime}+d^{\prime \prime}, d^{\prime} \in \mathcal{D}\left(S^{\prime}\right)$ and $d^{\prime \prime} \in \mathcal{D}\left(S^{\prime \prime}\right)$. From the smoothness of $f_{\bar{i}}^{*}(\cdot)$, we have $\left[f_{\bar{i}}^{*}\right]^{\prime}\left(x ; d_{\bar{i}}^{\prime}+d_{\bar{i}}^{\prime \prime}\right)=\left[f_{\bar{i}}^{*}\right]^{\prime}\left(x ; d_{\bar{i}}^{\prime}\right)+\left[f_{\bar{i}}^{*}\right]^{\prime}\left(x ; d_{\bar{i}}^{\prime \prime}\right)$, which gives (3.1) as needed.

Proposition 3.4. Property (b) in Theorem 3.2 is satisfied if

1. For all $n \geq 0$ and $y$, the condition $G^{\prime}(y ; d) \geq 0$ for all $d \in \mathcal{D}\left(S_{n}\right)$ implies the existence of KKT multipliers of

$$
\begin{aligned}
& \max _{y_{i}^{\prime} \in X, i \in S_{n}}-\sum_{i \in S_{n}} f_{i}^{*}\left(y_{i}^{\prime}\right) \\
& \text { s.t. } \quad \sum_{i \in S_{n}} y_{i}^{\prime}=\sum_{i \in S_{n}} y_{i}
\end{aligned}
$$

at a maximizer $\bar{y}$. Specifically, there exists $x \in X$ such that $x \in \partial f_{i}^{*}\left(\bar{y}_{i}\right)$ for all $i \in S_{n}$.

2. For every $\bar{i}, \bar{j} \in \mathcal{V}$, we can find a sequence of sets $\left\{\tilde{S}_{k}\right\}_{k=1}^{K} \subset\left\{S_{n+r}\right\}_{r=1}^{T}$ such that $\bar{i} \in \tilde{S}_{1}, \bar{j} \in \tilde{S}_{K}$, and $\tilde{S}_{k} \cap \tilde{S}_{k+1} \cap \mathcal{V}_{s m} \neq \emptyset$ for all $k \in\{1, \ldots, K-1\}$.

Proof. Recall the $y$ in property (b) in Theorem 3.2. Through condition (2), it suffices to prove that if $\tilde{S}_{1}$ and $\tilde{S}_{2}$ are such that $\tilde{S}_{1} \cap \tilde{S}_{2} \cap \mathcal{V}_{s m} \neq \emptyset$, then there exists $x$ such that $x \in \partial f_{i}^{*}\left(y_{i}\right)$ for all $i \in \tilde{S}_{1} \cup \tilde{S}_{2}$, which is in turn easy from condition (1). In Example 3.1, we see that $2 \notin \mathcal{V}_{s m}$, so Theorem 3.2 does not apply. 
3.1. Connection between Sections 2 and 3 . We now give a connection between the algorithms in the Sections 2 and 3. For a graph $(\mathcal{V}, \mathcal{E})$, construct the graph $\left(\mathcal{V}^{+}, \mathcal{E}^{+}\right)$via

$$
\begin{aligned}
& \mathcal{V}^{+}=\mathcal{V} \times\{0,1\} \\
& \mathcal{E}^{+}=\{((i, 0),(j, 0)):(i, j) \in \mathcal{E}\} \cup\{((i, 0),(i, 1)): i \in \mathcal{V}\} .
\end{aligned}
$$

One can easily check that $\left|\mathcal{V}^{+}\right|=2|\mathcal{V}|$ and $\left|\mathcal{E}^{+}\right|=|\mathcal{E}|+|\mathcal{V}|$. Let the function associated with the vertex $(i, s)$ be $f_{i, s}: X \rightarrow \overline{\mathbb{R}}$ defined by

$$
f_{(i, s)}(x)= \begin{cases}\frac{1}{2}\left\|x-\left[x_{0}\right]_{i}\right\|^{2} & \text { if } s=0 \\ f_{i}(x) & \text { if } s=1 .\end{cases}
$$

Note that $\min _{x \in X} \sum_{(i, s) \in \mathcal{V}^{+}} f_{(i, s)}(x)$ is equivalent to the problem (1.6) considered in Dykstra's algorithm.

The dual problem

$$
\max _{y_{(i, s)} \in X,(i, s) \in \mathcal{V}^{+}}\left\{-\sum_{(i, s) \in \mathcal{V}^{+}} f_{(i, s)}^{*}\left(y_{(i, s)}\right): \sum_{(i, s) \in \mathcal{V}^{+}} y_{(i, s)}=0\right\}
$$

(recall how (1.4) is derived as the dual of (1.3)) can be simplified to be

$$
\begin{aligned}
\max _{y_{i, s} \in X: i \in \mathcal{V}, s \in\{0,1\}}- & -\sum_{i \in \mathcal{V}} f_{i}^{*}\left(y_{i, 1}\right)-\sum_{i \in \mathcal{V}}\left[\frac{1}{2}\left\|y_{i, 0}+\left[x_{0}\right]_{i}\right\|^{2}-\frac{1}{2}\left\|\left[x_{0}\right]_{i}\right\|^{2}\right] \\
\text { s.t. } & \sum_{i \in \mathcal{V}}\left(y_{i, 0}+y_{i, 1}\right)=0 .
\end{aligned}
$$

Recall the dual problem in (1.7) and (1.8). Define the variable $z_{e} \in X^{|\mathcal{V}|}$ to be

$$
z_{e}:=\sum_{(i, j) \in \mathcal{E}} z_{(i, j)} .
$$

Suppose $z_{(i, j)} \in H_{(i, j)}^{\perp}$. Then $\delta_{H_{(i, j)}}^{*}\left(z_{(i, j)}\right)=0$. Also, $z_{(i, j)} \in H_{(i, j)}^{\perp} \subset D^{\perp}$, so $z_{e} \in D^{\perp}$. The dual problem in (1.7) and (1.8) becomes

$$
\max _{\left\{z_{e} \in D^{\perp}\right\} \cup\left\{z_{\alpha} \in X^{|\mathcal{V}|}: \alpha \in \mathcal{V}\right\}}-\sum_{i \in \mathcal{V}} \mathbf{f}_{i}^{*}\left(z_{i}\right)-\frac{1}{2}\left\|\sum_{i \in \mathcal{V}} z_{i}+z_{e}-x_{0}\right\|^{2}+\frac{1}{2}\left\|x_{0}\right\|^{2} .
$$

We now show how (3.9) and (3.10) are related.

Proposition 3.5. Consider the problems (3.9) and (3.10).

1. The two problems are related through a change of variables. Specifically, if $\left\{y_{(i, s)}\right\}_{(i, s) \in \mathcal{V}+} \subset X$ were obtained from $\left\{z_{i}\right\}_{i \in \mathcal{V}} \subset X^{|\mathcal{V}|}$ and $z_{e} \in X^{|\mathcal{V}|}$ by

$$
y_{i, 1}=\left[z_{i}\right]_{i} \text { and } y_{i, 0}=-\left[z_{e}\right]_{i}-\left[z_{i}\right]_{i} \text { for all } i \in \mathcal{V},
$$

then the objective values in (3.10) and (3.9) coincide. Conversely, if $\left\{z_{i}\right\}_{i \in \mathcal{V}}$ and $z_{e}$ were obtained from $\left\{y_{(i, s)}\right\}_{(i, s) \in \mathcal{V}+}$ by

$$
\left[z_{i}\right]_{j}=\left\{\begin{array}{ll}
0 & \text { if } j \neq i \\
y_{i, 1} & \text { if } j=i
\end{array} \text { and }\left[z_{e}\right]_{i}=-y_{i, 0}-y_{i, 1} \text { for all } i, j \in \mathcal{V},\right.
$$

then $z_{e} \in D^{\perp}$ and the objective values in (3.10) and (3.9) coincide. 
2. Let $S^{1} \subset \mathcal{V}^{+}$be a connected subset of vertices in the graph $\left(\mathcal{V}^{+}, \mathcal{E}^{+}\right)$so that $\left|S^{1}\right|>1$. Define $\Pi_{0} S^{1} \subset \mathcal{V}$ to be the set

$$
\Pi_{0} S^{1}:=\left\{i \in \mathcal{V}:(i, 0) \in S^{1}\right\} .
$$

Let $\Pi_{1} S^{1}$ be similarly defined. With respect to the graph $(\mathcal{V}, \mathcal{E})$, suppose that there is a subset $\mathcal{E}^{\prime}$ of $\mathcal{E}$ not containing any cycles that connects all the vertices in $\Pi_{0} S^{1}$. Since $S^{1}$ is a subset of connected vertices in the graph $\left(\mathcal{V}^{+}, \mathcal{E}^{+}\right)$, we have $\Pi_{1} S^{1} \subset \Pi_{0} S^{1}$.

Suppose that for a fixed $\left\{y_{(i, s)}\right\}_{(i, s) \in \mathcal{V}^{+}} \subset X$, a subproblem of (3.9) is solved with only variables $y_{(i, s)}$ indexed by $S^{1}$ allowed to vary while the other variables stay fixed. Then under the change of variables in (1), this subproblem is equivalent to solving the subproblem in (3.10) where

(a) $\left[z_{e}\right]_{i}$ is allowed to vary if and only if $i$ is an endpoint of some edge in $\mathcal{E}^{\prime}$, and

(b) $z_{i}$ is allowed to vary if and only if $i \in \Pi_{1} S^{1}$.

Proof. Statement 1 is obvious from the constructions.

We now work on Statement 2. From $\left|S^{1}\right|>1$ and the definition of $\left(\mathcal{V}^{+}, \mathcal{E}^{+}\right)$, if $(i, 1) \in \mathcal{V}^{+}$, then $(i, 0) \in \mathcal{V}^{+}$. So for each $i \in \mathcal{V}$, there are three cases: $(1)(i, 1) \notin \mathcal{V}^{+}$ and $(i, 0) \notin \mathcal{V}^{+}$(in which case there is nothing to do), (2) $(i, 0) \in \mathcal{V}^{+}$and $(i, 1) \notin \mathcal{V}^{+}$ and $(3)(i, 0) \in \mathcal{V}^{+}$and $(i, 1) \in \mathcal{V}^{+}$.

In case (2), if the term $y_{i, 0}$ is in $S^{1}$, then $y_{i, 0}$ affects only the $\frac{1}{2}\left\|y_{i, 0}+\left[x_{0}\right]_{i}\right\|^{2}$ in (3.9). In turn, $\left[z_{e}\right]_{i}$ only affects $\frac{1}{2}\left\|\left[z_{e}\right]_{i}+\left[z_{i}\right]_{i}-\left[x_{0}\right]_{i}\right\|^{2}$ in the quadratic term in (3.10).

In case (3), it is clear that the term $f_{i}\left(y_{i, 1}\right)$ varies through $y_{i, 1}$ if and only if $\mathbf{f}_{i}\left(z_{i}\right)$ varies through $z_{i}$. Recall that $\Pi_{0} S^{1} \subset \Pi_{1} S^{1}$, so if the term $y_{i, 1}$ is in $S^{1}$, then $y_{i, 0}$ is in $S^{1}$. The terms $y_{i, 1}$ and $y_{i, 0}$ then combine to affect $f_{i}^{*}\left(y_{i, 1}\right)+\frac{1}{2}\left\|y_{i, 0}+\left[x_{0}\right]_{i}\right\|^{2}$. Correspondingly, $\left[z_{e}\right]_{i}$ and $\left[z_{i}\right]_{i}$ combine to affect $\mathbf{f}_{i}^{*}\left(z_{i}\right)+\frac{1}{2}\left\|\left[z_{e}\right]_{i}+\left[z_{i}\right]_{i}-\left[x_{0}\right]_{i}\right\|^{2}$.

To wrap up, note that the constraint in (3.9) corresponds to $z_{e} \in D^{\perp}$.

One can easily figure out that (2a) in Proposition 3.5 corresponds to varying $z_{(i, j)}$ for all $(i, j) \in \mathcal{E}^{\prime}$ in the original dual problem of (1.7) and (1.8).

4. Accelerated methods for (1.7). In this section, we write down an accelerated proximal gradient (APG) algorithm [34, 7, 45] on the dual problem described through (1.7) and (1.8) that allows for greedy steps that can be performed asynchronously. Before we continue, we remark that we had shown that an APG with greedy steps can be performed on the formulation (1.10) in [37]. We point out that the APG derived from (1.7) and (1.8) has a much lower Lipschitz constant and allows for greedy steps of the form (2.3).

We first recall a variant of the accelerated proximal gradient in [45]. In view of the clash of variables, we substitute the variables $x, y$ and $z$ in [45] to be $u, v$ and $w$, and then substitute the $f^{P}(\cdot)$ in [45] for the function $-F(\cdot)$ in (1.8). (Their algorithm includes allowing for the domain of the optimization problem for $w^{k+1}$ to change in each iteration, which we omit.) Let

$$
\begin{aligned}
l(u, v)= & \frac{1}{2}\left\|x_{0}-\sum_{\alpha \in \mathcal{E} \cup \mathcal{V}} v_{\alpha}\right\|^{2}-\frac{1}{2}\left\|x_{0}\right\|^{2} \\
& +\underbrace{\left\langle\left(\sum_{\alpha \in \mathcal{E} \cup \mathcal{V}} v_{\alpha}\right)-x_{0}, \sum_{\alpha \in \mathcal{E} \cup \mathcal{V}}\left(u_{\alpha}-v_{\alpha}\right)\right\rangle}+\underbrace{\sum_{\alpha \in \mathcal{E} \cup \mathcal{V}} h_{\alpha}\left(u_{\alpha}\right)}
\end{aligned}
$$


Remark 4.1. We now explain the formula $l(u, v)$ above. In [45], the function that Algorithm 4.1 aims to minimize was $f^{P}(u)=f(u)+P(u)$, where $f(\cdot)$ is smooth and $P(\cdot)$ admits an easy calculation of its proximal, and has a linearization $f(v)+$ $\langle\nabla f(v), u-v\rangle+P(u)$. The underbraced terms in (4.1) play the role of the terms $f(v)$, $\langle\nabla f(v), u-v\rangle$ and $P(u)$ in the linearization of $-F(u)$ in (1.8).

\section{Algorithm 4.1 From [45, Algorithm 1]}

We want to find $u$ to minimize $-F(\cdot)$, which is the sum of a convex smooth function and a convex separable function. Choose $\theta_{0} \in(0,1], u^{0}, w^{0} \in \operatorname{dom}(P)$. Let $L>0$ be such that

$$
l(u, v)+\frac{L}{2}\|u-v\|^{2} \geq-F(u) \text { for all } u, v \in\left[X^{|\mathcal{V}|}\right]^{|\mathcal{V} \cup \mathcal{E}|} .
$$

Go to 1 .

1. Let

$$
\begin{aligned}
v^{k} & =\left(1-\theta_{k}\right) u^{k}+\theta_{k} w^{k} \\
w^{k+1} & \left.=\underset{u \in X}{\arg \min }\left\{l\left(u ; v^{k}\right)+\theta_{k} L \frac{1}{2}\left\|u-w^{k}\right\|^{2}\right)\right\}, \\
\hat{u}^{k+1} & =\left(1-\theta_{k}\right) u^{k}+\theta_{k} w^{k+1},
\end{aligned}
$$

Choose $u_{k+1}$ to be such that

$$
-F\left(u^{k+1}\right) \leq l\left(\hat{u}^{k+1} ; v^{k}\right)+\frac{L}{2}\left\|\hat{u}^{k+1}-v^{k}\right\|^{2} .
$$

Choose $\theta_{k+1} \in(0,1]$ satisfying

$$
\frac{1-\theta_{k+1}}{\theta_{k+1}^{2}} \leq \frac{1}{\theta_{k}^{2}}
$$

$k \leftarrow k+1$, and go to 1 .

Remark 4.2. Algorithm 4.1 requires the condition (4.2). Since $l(\cdot, v)$ is the linearization of $-F(\cdot)$ at $v$, we have $l(v, v)=-F(v)$. Since the smooth portions of both $l(u, v)+\frac{L}{2}\|u-v\|^{2}$ and $-F(\cdot)$ are quadratics, showing (4.2) is equivalent to finding $L>0$ such that the Hessian of the smooth portion of $l(u, v)+\frac{L}{2}\|u-v\|^{2}$ is greater than that of $-F(\cdot)$, i.e.,

$$
\frac{L}{2} \sum_{\alpha \in \mathcal{E} \cup \mathcal{V}}\left\|u_{\alpha}\right\|^{2} \geq \frac{1}{2}\left\|\sum_{\alpha \in \mathcal{E} \cup \mathcal{V}} u_{\alpha}\right\|^{2} .
$$

Since the variables $\left\{u_{\alpha}\right\}_{\alpha \in \mathcal{E} \cup \mathcal{V}}$ are to satisfy the sparsity pattern in Proposition 2.7 , we show that $L$ can be chosen as follows.

Proposition 4.3. (Choice of $L$ in Algorithm 4.1) In order to satisfy (4.4) while obeying the sparsity pattern in Proposition 2.7, we can choose $L$ to be $\bar{d}+1$, where $\bar{d}$ is the maximum degree of the vertices in the graph.

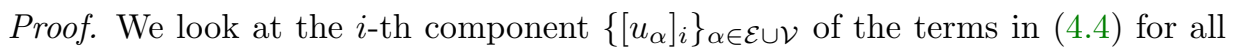


$i \in \mathcal{V}$. As long as we can prove that

$$
\frac{\bar{d}+1}{2} \sum_{\alpha \in \mathcal{E} \cup \mathcal{V}}\left\|\left[u_{\alpha}\right]_{i}\right\|^{2} \geq \frac{1}{2}\left\|\sum_{\alpha \in \mathcal{E} \cup \mathcal{V}}\left[u_{\alpha}\right]_{i}\right\|^{2}
$$

the conclusion will follow. In view of the sparsity pattern of the $u$ 's in Proposition 2.7 , most of the $\left[u_{\alpha}\right]_{i}$ 's are zero. For all $i \in \mathcal{V}$, we define $\tilde{\mathcal{E}}_{i}$ to be the set of all edges $e \in \mathcal{E}$ such that one of the endpoints is $i$. Then (4.5) reduces to

$$
\frac{\bar{d}+1}{2}\left(\left\|\left[u_{i}\right]_{i}\right\|^{2}+\sum_{e \in \tilde{\mathcal{E}}_{i}}\left\|\left[u_{e}\right]_{i}\right\|^{2}\right) \geq \frac{1}{2}\left\|\left[u_{i}\right]_{i}+\sum_{e \in \tilde{\mathcal{E}}_{i}}\left[u_{e}\right]_{i}\right\|^{2},
$$

We form the vector $\tilde{u} \in X^{\left|\tilde{\mathcal{E}}_{i}\right|+1}$ so that it contains $\left[u_{i}\right]_{i}$ and $\left\{\left[u_{e}\right]_{i}\right\}_{e \in \tilde{\mathcal{E}}_{i}}$ as its components. The formula (4.6) can be seen to be equivalent to

$$
\tilde{u}^{T}\left[\left(\begin{array}{cccc}
(\bar{d}+1) I & & & \\
& (\bar{d}+1) I & & \\
& & \ddots & \\
& & & (\bar{d}+1) I
\end{array}\right)-\left(\begin{array}{cccc}
I & I & \cdots & I \\
I & I & \cdots & I \\
\vdots & \vdots & \ddots & \vdots \\
I & I & \cdots & I
\end{array}\right)\right] \tilde{u} \geq 0
$$

which is clearly true. Thus we are done.

If we had used the formulation in (2.3) without exploiting the sparsity in Proposition 2.7, then the corresponding $L$ would be $|\mathcal{V} \cup \mathcal{E}|$, which is a much larger number than $\bar{d}+1$ in most large graphs. Recall that $L$ was chosen so that (4.2) holds, and $L$ should be as small as possible subject to this condition so that the step for calculating $w^{k+1}$ in Algorithm 4.1 would be minimizing a function closer to $-F(\cdot)$. This lower value of $\bar{d}+1$ is one advantage of applying the APG on the dual problem from (1.7) and (1.8).

We recall the convergence result of Algorithm 4.1.

Theorem 4.4. [45, Corollary 1] (Convergence of Algorithm 4.1) Let

$$
\left\{\left(u^{k}, v^{k}, w^{k}, \theta_{k}, X_{k}\right)\right\}_{k}
$$

be generated by Algorithm 4.1 with $\theta_{0}=1$. For any $\epsilon>0$. Suppose $\theta_{k} \leq \frac{2}{k+2}$. Then for any $u \in \operatorname{dom}(P)$ with $-F(u) \leq \inf F+\epsilon$, we have

$$
\min _{i=0,1 \ldots, k+1}\left\{-F\left(u^{i}\right)\right\} \leq-F(u)+\epsilon \text { whenever } k \geq \sqrt{\frac{4 L}{\epsilon}}\left\|x-x^{0}\right\|-2 .
$$

In the particular case where there is a minimizer $u^{*}$, Theorem 4.4 says that an $\epsilon$-optimal solution for $-F(\cdot)$ is obtained if the number of iterations $k$ is the Nesterov accelerated rate of $O\left(\sqrt{\frac{1}{\epsilon}}\right)[34,7,45]$. In the case of Dykstra's algorithm (or block coordinate minimization), the number of iterations needed to obtain an $\epsilon$-optimal solution is typically $O\left(\frac{1}{\epsilon}\right)$ (see for example $[8,6]$ ), which is slower than the $O\left(\sqrt{\frac{1}{\epsilon}}\right)$ rate.

Remark 4.5. (On Theorem 4.4) The proof of Theorem 4.4 in [45] is for the algorithm with a modified (4.3), with the left hand side being $l\left(u^{k+1} ; v^{k}\right)+\frac{L}{2}\left\|u^{k+1}-v^{k}\right\|^{2}$ instead. But the proof in [45] carries over with no changes at all.

We now elaborate on how the greedy step can be applied to Algorithm 4.1. 
Remark 4.6. (Greedy steps in Algorithm 4.1) We remark that the greedy step can be performed in (4.3). Note that $u^{k+1}$ in (4.3) can be chosen to be $\hat{u}^{k+1}$. But the greedy steps of the form (2.3) can be performed in (4.3) (with $u$ 's in place of $z$ 's there). These greedy steps can be performed asynchronously like as discussed in Remark 2.11.

5. Conclusion. We have done what we set out to do in Subsection 1.3. In short, we noticed that a dual ascent algorithm can give us a distributed and asynchronous algorithm with deterministic convergence for time-varying graphs when the function on each vertex is strongly convex with a known modulus. A separate related algorithm is proposed for the case when the function on each vertex is not necessarily strongly convex, but Example 3.1 shows that the algorithm can fail to converge.

Note that in Example 3.1, the failure of Algorithm 3.1 is identified by the primal variable -1 in $\partial f_{1}^{*}(0)$ and $\partial f_{2}^{*}(0)$ being obtained when $S=\{1,2\}$, while the primal variable 1 in $\partial f_{2}^{*}(0)$ and $\partial f_{3}^{*}(0)$ was obtained when $S=\{2,3\}$. Is there a primal dual algorithm that has a number of the properties listed in Subsection 1.1 and 1.3?

Acknowledgments. We thank Mert Gürbüzbalaban and Necdet Serhat Aybat for discussions leading to this paper. Lastly, we thank the associate editor, the anonymous referees and the journal staff for the quick review of this paper.

\section{REFERENCES}

[1] F. Abboud, E. Chouzenoux, J.-C. Pesquet, J.-H. Chenot, and L. Laborelli, Dual blockcoordinate forward-backward algorithm with application to deconvolution and deinterlacing of video sequences, Journal of Mathematical Imaging and Vision, 59 (2017), pp. 415-431.

[2] N. Aybat and E. Hamedani, A primal-dual method for conic constrained distributed optimization problems, in Advances in Neural Information Processing Systems 29, Curran associates, Red Hook, NY, 2016, pp. 5049-5057.

[3] A. Aytekin, H. Feyzmahdavian, and M. Johansson, Analysis and implementation of an asynchronous optimization algorithm for the parameter server. arxiv eprint 1610.05507, 2016.

[4] H. Bauschke And J. Borwein, On projection algorithms for solving convex feasibility problems, SIAM Rev., 38 (1996), pp. 367-426.

[5] H. Bauschke and P. Combettes, Convex Analysis and Monotone Operator Theory in Hilbert Spaces, Springer, 2011.

[6] A. BECK, On the convergence of alternating minimization for convex programming with applications to iteratively reweighted least squares and decomposition schemes, SIAM J. Optim., 25 (2015), pp. 185-209.

[7] A. Beck And M. Teboulle, A fast iterative shrinkage-thresholding algorithm for linear inverse problems, SIAM J. Imaging Sciences, 2 (2009), pp. 183-202.

[8] A. Beck and L. Tetruashvili, On the convergence of block coordinate descent type methods, SIAM J. Optim., 23 (2013), pp. 2037-2060.

[9] P. Bianchi, W. Hachem, and F. Iutzeler, A stochastic coordinate descent primal-dual algorithm and applications, in 2014 IEEE International workshop on machine learning for signal processing, Sept. 21-24 2014.

[10] S. Boyd, A. Ghosh, B. Prabhakar, and D. Shah, Randomized gossip algorithms, IEEE Trans. Information Theory, 52 (2006), pp. 2508-2530.

[11] S. Boyd, N. Parikh, E. Chu, B. Peleato, and J. Eckstein, Distributed optimization and statistical learning via the alternating direction method of multipliers, Foundations and Trends in Machine Learning, 3 (2010), pp. 1-122.

[12] J. Boyle And R. Dykstra, A method for finding projections onto the intersection of convex sets in Hilbert spaces, in Advances in Order Restricted Statistical Inference, Lecture notes in Statistics, Springer, New York, 1985, pp. 28-47.

[13] L. Cannelli, F. Facchinei, V. Kungurtsev, and G. Scutari, Asynchronous parallel algorithms for nonconvex big-data optimization, part I: Model and convergence. arxiv eprints 1607.04818, 2016. 
[14] P. Combettes, D. DŨng, And B. Vũ, Dualization of signal recovery problems, Set-Valued and Variational Analysis, 18 (2010), pp. 373-404.

[15] P. Combettes, D. Dũng, And B. Vũ, Proximity for sums of composite functions, Journal of Mathematical Analysis and Applications, 380 (2011), pp. 680-688.

[16] P. Combettes And J. ECKstein, Asynchronous block-iterative primal-dual decomposition methods for monotone inclusions, Math. Prog. Ser. B, 168 (2018), pp. 645-672.

[17] L. Condat, A primal-dual splitting method for convex optimization involving Lipschitzian, proximable and linear composite terms, Journal of Optimization Theory and Applications, 158 (2013), pp. 460-479.

[18] F. Deutsch, Best Approximation in Inner Product Spaces, Springer, 2001. CMS Books in Mathematics.

[19] A. G. Dimakis, S. Kar, J. M. F. Moura, M. G. Rabbat, and A. Scaglione, Gossip algorithms for distributed signal processing, Proceedings of the IEEE, 98 (2010), pp. 1847-1864.

[20] R. Dykstra, An algorithm for restricted least-squares regression, J. Amer. Statist. Assoc., 78 (1983), pp. 837-842.

[21] J. ECKSTEIn And B. SVAiter, General projective splitting methods for sums of maximal monotone operators, SIAM J. Control Optim., 48 (2009), pp. 787-811.

[22] R. Escalante and M. Raydan, Alternating Projection Methods, SIAM, 2011.

[23] D. GABAY, Chapter IX Applications of the method of multipliers to variational inequalities, in Augmented Lagrange Methods: Applications to the Solution of Boundary Valued Problems, M. Fortin and R. Glowinski, eds., vol. 15, North Holland, Amsterdam, 1983, pp. 299331.

[24] N. Gaffke and R. Mathar, A cyclic projection algorithm via duality, Metrika, 36 (1989), pp. 29-54.

[25] M. Gurbuzbalaban, A. Ozdaglar, and P. Parrilo, On the convergence rate of incremental aggregated gradient algorithms, SIAM J. Optim., 27 (2017), pp. 1035-1048.

[26] S. Han, A successive projection method, Math. Programming, 40 (1988), pp. 1-14.

[27] H. Hundal and F. Deutsch, Two generalizations of Dykstra's cyclic projections algorithm, Math. Programming, 77 (1997), pp. 335-355.

[28] F. Iutzeler, P. Bianchi, P. Ciblat, and W. Hachem, Asynchronous distributed optimization using a randomized alternating direction method of multipliers, in Proceedings of the 52nd Conference on Decision Control, Florence, Italy, Dec. 2013, pp. 3671-3676.

[29] S. LeE And A. Nedić, Distributed random projection algorithm for convex optimization, IEEE Journal of Selected Topics in Signal Processing, 7 (2013), pp. 221-229.

[30] A. NEDICH, Convergence rate of distributed averaging dynamics and optimization in networks, Foundations and Trends in Systems and Control, 2 (2015), pp. 1-100.

[31] A. NEDICH, Fast algorithms for distributed optimization over time-varying graphs. Talk at DIMACS Workshop on Distributed Optimization, Information Processing, and Learning, https://www.nrel.gov/grid/assets/pdfs/aeg-nedich.pdf, 2017.

[32] A. Nedich And A. Olshevsky, Distributed optimization over time-varying directed graphs, IEEE Transactions on Automatic Control, 60 (2015), pp. 601-615.

[33] A. Nedich, A. Olshevsky, And W. Shi, Achieving geometric convergence for distributed optimization over time-varying graphs, SIAM J. Optim., 27 (2017), pp. 2597-2633.

[34] Y. Nesterov, A method for solving a convex programming problem with rate of convergence $O\left(\frac{1}{k^{2}}\right)$, Soviet Math. Doklady, 269 (1983), pp. 543-547. (in Russian).

[35] I. Notarnicola and G. Notarstefano, Asynchronous distributed optimization via randomized dual proximal gradient, IEEE Transactions on Automatic Control, 62 (2017), pp. 20952106.

[36] A. Ozdaglar, A. Nedić, And P. Parrilo, Constrained consensus and optimization in multiagent networks, IEEE Trans. Automat. Control, 55 (2010), pp. 922-938.

[37] C. PANG, The supporting halfspace - quadratic programming strategy for the dual of the best approximation problem, SIAM J. Optim., 26 (2016), pp. 2591-2619.

[38] C. PANG, Dykstra splitting and an approximate proximal point algorithm for minimizing the sum of convex functions, (2017). arxiv eprint 1709.09499.

[39] Z. Peng, Y. Xu, M. Yan, And W. Yin, AROCK: An algorithmic framework for asynchronous parallel coordinate updates, SIAM J. Sci. Comput., 38 (2016), pp. A2851-A2879.

[40] S. Phade and V. Borkar, A distributed Boyle-Dykstra-Han scheme, SIAM J. Optim., 27 (2017), pp. 1880-1897.

[41] S. S. Ram, A. Nedić, And V. Veeravalli, Distributed stochastic subgradient projection algorithms for convex optimization, J. Optim. Theory and Appl., 147 (2010), pp. 516-545.

[42] W. ShI, Q. Ling, G. WU, AND W. YIN, EXTRA: An exact first-order algorithm for decentralized consensus optimization, SIAM J. Optim., 25 (2015), pp. 944-966. 
[43] R. Tibshirani, Dykstra's algorithm, ADMM, and coordinate descent: Connections, insights, and extensions, (2017).

[44] P. Tseng, Convergence of a block coordinate descent method for nondifferentiable minimization, J. Optim. Theory Appl., 109 (2001), pp. 475-494.

[45] P. Tseng, On accelerated proximal gradient methods for convex-concave optimization, (2008). manuscript.

[46] B. VŨ, A splitting algorithm for dual monotone inclusions involving cocoercive operators, Advances in Computational Mathematics, 38 (2013), pp. 667-681. 